\title{
Statistical Inferences of Type-II Progressively Hybrid Censored Fuzzy Data with Rayleigh Distribution
}

\author{
Ankita Chaturvedi \\ Banaras Hindu University
}

\author{
Sanjay Kumar Singh \\ Banaras Hindu University
}

\author{
Umesh Singh \\ Banaras Hindu University
}

\begin{abstract}
This article presents the procedures of parameter estimation based on Type-II progressively hybrid censored fuzzy lifetime data. Classical as well as the Bayesian procedures for the estimation of unknown model parameters has been developed. For this purpose we have considered the problem of point estimation of the parameter of Rayleigh distribution. The estimators obtained here are Maximum likelihood (ML) estimator, Method of moments (MM) estimator, Computational approach (CA) estimator and Bayes estimator. Highest posterior density (HPD) credible intervals of the unknown parameter are obtained by using Markov Chain Monte Carlo (MCMC) technique. For numerical illustration, a real data set has been considered.
\end{abstract}

Keywords: Type-II progressive hybrid censoring, fuzzy lifetime data, Rayleigh distribution, maximum likelihood (ML) estimator, method of moments (MM) estimator, computational approach (CA) estimator.

\section{Introduction}

In a life testing and reliability experiment, several items are put on test and the experiment is terminated when all of them fail to work. This process is often very time-consuming and costly. Besides this, situations do arise when one cannot observe the complete life time of all the items put on test. For example, units may be taken out of the study before completion of experiment because it is damaged due to some reasons or lack of money compels to terminate the experiment prior to its completion. Data obtained from such experiments are called censored data because in such cases one is not completely ignorant about the lifetime of the censored items, in the sense that a partial information about their lifetime is known. The scheme of taking out items from the experiment is called censoring scheme. The censoring schemes arise either in a natural way in life test experiments or deliberately implemented in the experiment due to the various constraints imposed on the experimentation. In present day scenario, censoring is a need to reduce total time and cost associated with the life-testing experiment.

Various censoring schemes are available in the literature to cope up with the need of the investigator and constraints on the experiment. Each of them has their own merits and de- 
merits. A situation which is often met in some of the life testing experiment, particularly in those experiments where costly items are put to test, is that units are removed from the experiment before the final termination of the experiment i.e. removal of items are allowed prior to completion of the experiment and such type of scheme is known as progressive censoring scheme. A censoring scheme which is getting extremely popular nowadays is Type-II progressive censoring scheme. It was introduced by Cohen (1965). Since then, many authors including Cohen and Norgaard (1977), Davis and Feldstein (1979), Viveros and Balakrishnan (1994), Balakrishnan and Sandhu (1995), Krishna and Kumar (2013), Singh, Singh, and Kumar (2013) have discussed the estimation procedures and applications of progressive censored sample for various lifetime models. An extensive review of the literature on progressive censoring can be seen from Balakrishnan and Aggarwala (2000) and Balakrishnan (2007). This censoring scheme allows removals within Type-II censoring scheme in the following way. Suppose $\mathrm{n}$ items are put simultaneously on test at time $t=0$ and it is decided to observe complete lifetime of exactly $m(<n)$ items. In addition to it, $\mathrm{m}$ other integers $R_{1}, R_{2}, \ldots, R_{m}$ such that $R_{1}+R_{2}+\cdots+R_{m}+m=n$ are also specified with the purpose that at the time of the first failure, $R_{1}$ items will be randomly removed from the experiment and the experiment will continue with the remaining $\left(n-R_{1}-1\right)$ items. Similarly, at the time of the second failure, $R_{2}$ items will be randomly removed from the experiment. This process will be repeated at each failure and finally, at the time of $m^{\text {th }}$ failure all of the surviving items (i.e. $R_{m}$ items) will be removed from the experiment.

Nowadays, high-quality items are being produced due to advancement in the technology hence a major criticism being faced by Type-II progressive censoring scheme is regarding the total time and cost (which may be very large) associated with such life-testing experiment. Keeping this point in mind, Kundu and Joarder (2006) proposed a new censoring scheme which is a mixture of hybrid and Type-II progressive censoring schemes and named it as Type-II progressive hybrid censoring which ensures that the experiment time can not exceed a prefixed time T; see also Childs, Chandrasekar, and Balakrishnan (2008), Kundu (2007), Kundu, Joarder, and Krishna (2009).

It is important to note here that most of the statistical procedures mentioned above are developed considering that all the observations are available in most precise form. But in real life situations, it is not always possible to obtain the experimental observations in precise form due to experimental errors, human errors, precision of measurement and several other practical difficulties. Such type of imprecision in the data may creep in due to linguistic descriptions also; see Zadeh (1975). For example, if we want to know failure time of an item in survival analysis it may be reported as "approximately between 99 hours to 101 hours", "about 100 hours" etc. In life testing and reliability problems, the variable of interest is continuous, while in the process of data collection, we consider that all measurements are in precise numbers which looks illogical in the sense that it is impractical to record an observation of a continuous variable in precise number. In this way, some error is incorporated in the observed data during the measurement and hence make these imprecise. For example, suppose exact failure time of an item in survival analysis is reported as 100 hours. It can be argued that no value can be measured as absolutely 100. There will be always a difference between true and reported value and hence the reported value is an approximate value. In fact, it corresponds to any value, between 99 hours to 101 hours i.e. more than 99 hours but less than 101 hours. To deal with such type of imprecise data, which arises due to linguistic variability and error in measurement; classical estimation methods are not appropriate. However, imprecision in the data can be easily encoded with the help of fuzzy numbers and then fuzzy set theory provides an appropriate tool to quantify the uncertainty due to such ambiguities.

From the above discussion, it is clear that the idea of formulation of measurement error in terms of fuzzy set theory looks more reasonable because it takes care of the imprecision and uncertainty of observations as well as flexibility in truth values. The fuzzy approach relates to a grade of membership between $[0,1]$, defined in terms of the membership function of a fuzzy number and in a fuzzy set transition from false to true is gradual. On the other 
hand, conventional crisp sets (absolute true values) are defined by either membership or nonmembership and there is a sharp distinction or boundary between members and non-members of the set. Hence, fuzzy set modifies the classical notion of binary membership of crisp set to represent the uncertainty in data, a brief description of which is given below:

Theory of fuzzy sets was introduced by Zadeh (1965) and the Membership functions (MFs) form the base of fuzzy set theory. The membership function (MF) of a fuzzy set is nothing but the generalization of the indicator function in classical crisp sets hence we can graphically represent a fuzzy set with the help of its MF. The degree to which an object belongs to a fuzzy set is denoted by a membership value between 0 and 1 . Hence, MF associated with a given fuzzy set maps an input value to its appropriate membership value. In mathematical terminology a fuzzy number is a subset (say A), denoted by $\widetilde{A}$, of the set of real numbers $\mathbb{R}$ and is characterized by membership function $\xi_{\widetilde{A}}(x)$ which associates with each point in A, a real number in interval $[0,1]$, with the value of $\xi_{\widetilde{A}}(x)$ at $\mathrm{x}$ representing the grade of membership of $\mathrm{x}$ in A.

A priori information about the object under study is applied in form of MF to increase the accuracy. We are using MFs to code the observed data which is not in complex functional form hence use of complex functions do not add more precision. Therefore simple functions having natural interpretation are used to define a MF. In fact, the shapes of MFs are important for a particular problem since they have significant effect on a fuzzy inference system. The shapes of MFs may be taken as triangular, trapezoidal, Gaussian, etc. depending upon the nature of data. For any fuzzy number $\widetilde{A}$ there exist four numbers $a 1, a 2, a 3, a 4 \in \mathbb{R}$ and two functions $\beta_{\widetilde{A}}(x), \gamma_{\widetilde{A}}(x): \mathbb{R} \longrightarrow[0,1]$, where $\beta_{\widetilde{A}}(x)$ is non-decreasing and $\gamma_{\widetilde{A}}(x)$ is non-increasing, such that we can describe a membership function $\xi_{\widetilde{A}}(x)$ in the following manner:

$$
\xi_{\widetilde{A}}(x)=\left\{\begin{array}{lll}
0 & \text { if } & x<a 1 \\
\beta_{\widetilde{A}}(x) & \text { if } & a 1 \leq x<a 2 \\
1 & \text { if } & a 2 \leq x<a 3 \\
\gamma_{\widetilde{A}}(x) & \text { if } & a 3 \leq x<a 4 \\
0 & \text { if } & a 4<x
\end{array}\right.
$$

Most common fuzzy number is the "trapezoidal fuzzy number" whose both sides are linear. It is used to encode data such as "more or less between 99 and 100" or "approximately between 99 and 100". "Triangular fuzzy number" is a trapezoidal fuzzy number for which (a2=a3) and it is used to encode data such as "about 100" and "more or less 100". Similarly Triangular fuzzy numbers with only right side or left side is useful for situations such as "just before 100" or "just after 100" for which $(a 2=a 3=a 4)$ and $(a 1=a 2=a 3)$ respectively. If $a 1=a 2$ and $a 3=a 4$, then we get the rectangular fuzzy numbers, which may represent expressions such as, e.g. "between 99 and 101". In the case of $a 1=a 2=a 3=a 4=a$ we get a crisp number, i.e. a fuzzy number which is no longer vague but represents a precise value that can be identified with the proper real number $a$.

For further details about crisp and fuzzy sets see; Dubois (1980), Dubois and Prade (1998), Klir and Yuan (1995), Zimmermann (2000), Viertl (2011). Probability measure for fuzzy events was discussed by Zadeh (1968). Singpurwalla and Booker (2004) discussed membership functions and probability measure of fuzzy sets. Viertl (2009) have considered generalized parametric procedures for reliability characteristic including fuzzy point estimators and generalized Bayesian procedures. Huang, Zuo, and Sun (2006), Liu (2012), Cai (2012), Shafiq and Viertl $(2015,2017)$ have also discussed estimation of survival function and Hazard rate. Liu and Li (2012) have discussed two types of estimators for cumulative distribution function (CDF) based on fuzzy data. Recently Pak, Parham, and Saraj (2013a) discussed various classical and Bayesian methods of estimation for Weibull distribution when data is available in the form of fuzzy numbers. Also, Pak, Parham, and Saraj (2013b) proposed a new method to determine the maximum likelihood estimate of the scale parameter of a Rayleigh distribution under doubly Type-II censored sample from fuzzy data and Pak, Parham, and Saraj (2014) 
discussed inferences for the Rayleigh distribution based on progressive Type-II fuzzy censored data.

In the present article, we are assuming that we have only vague knowledge of the observed values of the variable because accurate measurement of variable is not possible, i.e. the investigator can provide only approximate lifetimes of the items as guess values only by specifying that the exact value lies in a small interval around the noted observed value. The interval is to formed by the investigator according to his belief in various value of the interval. For example, suppose that the least count of the measurement is $2 \mathrm{~h}$ units, then the interval can be formed by adding and subtracting h, from the noted observed lifetime of unit. Naturally, the value of the factor $h$ will vary from problem to problem depending upon its nature and extent of error in the observed data.

The objective of the present study is to discuss various methods of parameter estimation under Type-II progressive hybrid censoring scheme when the observations are provided in the form of fuzzy numbers. For this purpose, we have considered the problem of point estimation of the parameter of Rayleigh distribution since it is a popular model being used in different branches of science and engineering. It is frequently used in life testing experiments especially in reliability and survival analysis. It was proposed by Lord Rayleigh for the first time in 1880. Since then several authors have discussed its properties and have developed inferential procedures under different situations in verity of fields; for details see, Dyer and Whisenand (1973), Bhattacharya and Tyagi (1990), Chung (1995), Dey and Maiti (2012).

Rest of the paper is organized as follows: In section 2, model under present censoring scheme is discussed briefly. Methods of classical and Bayes estimation are discussed in section 3. It includes maximum likelihood estimation, method of moments estimation and computational approach estimation method. Further, Bayes estimator and HPD credible intervals are also obtained. Based on the numerical results of point estimation of parameter of Rayleigh distribution, performance of the estimators are compared in terms of mean squared error in section 4. Also a real data set is analyzed for illustrative purpose in this section. Finally, the concluding remarks are given in section 5 .

\section{Model description and notations}

Consider a life testing experiment in which $n$ identical items are put to test. Under the Type-II progressive hybrid censoring scheme, an integer $m<n$ is prefixed along with $T$ and integers $R_{1}, R_{2}, \ldots, R_{m}$ such that $R_{1}+R_{2}+\cdots+R_{m}+m=n$, where $n$ is the total number of items put on test, $m$ and $T$ are the prefixed maximum number of failure observations and maximum time for termination of the experiment respectively and $R_{1}, R_{2}, \ldots, R_{m}$ are the prefixed number of items to be removed from the experiment at 1st, 2nd,..., mth observed failures. Let $X_{1: m: n} \leq X_{2: m: n} \leq \cdots \leq X_{m: m: n}$ denote the $\mathrm{m}$ observed ordered failure times. Under this censoring scheme experiment is terminated at a random time $\min \left\{X_{m: m: n}, T\right\}$. That is, the experiment starts with $n$ items and at failure $X_{1: m: n}, R_{1}$ items are randomly removed from the experiment and experiment continues with the remaining $\left(n-R_{1}-1\right)$ items. At second failure $X_{2: m: n}, R_{2}$ items are randomly removed from the experiment. The experiment continues in this fashion removing the prefixed number of items from the experiment at each observed failure. If the $m$ th observed failure $X_{m: m: n}$ occurs before prefixed time $T$, the experiment stops at time $X_{m: m: n}$. Otherwise, the experiment is terminated at prefixed time $T$ where $X_{J: m: n} \leq T \leq X_{J+1: m: n}$, by removing all the remaining $R_{J}^{*}=n-\sum_{i=1}^{J} R_{i}-J$ surviving items. Thus, under Type-II progressive hybrid censoring scheme two cases arise.

Case I: If $X_{m: m: n}<T$ the observed life times would be $\left\{X_{1: m: n}, \ldots, X_{m: m: n}\right\}$

Case II: If $X_{J: m: n}<T<X_{J+1: m: n}$ the observed life times would be $\left\{X_{1: m: n}, \ldots, X_{J: m: n}\right\}$

Let $f(x)$ and $F(x)$ denotes the probability density function and cumulative distribution func- 
tion of the distribution under study respectively then following Childs, Chandrasekar, and Balakrishnan (2008), we can write the likelihood function for the above mentioned two cases as follows:

For case I when $X_{m: m: n}<T$, the likelihood function is given as follows:

$$
l(\vartheta)=B_{1} \prod_{i=1}^{m} f_{X}\left(x_{i: m: n} ; \vartheta\right)\left[1-F_{X}\left(x_{i: m: n} ; \vartheta\right)\right]^{R_{i}}
$$

Where $B_{1}=\prod_{i=1}^{m}\left[n-\sum_{k=1}^{i-1}\left(1+R_{k}\right)\right]$

For case II when $T<X_{m: m: n}$ and $X_{J: m: n}<T<X_{J+1: m: n}$, the likelihood function is given as follows:

$$
l(\vartheta)=B_{2} \prod_{i=1}^{J}\left[f_{X}\left(x_{i: m: n} ; \vartheta\right)\left\{1-F_{X}\left(x_{i: m: n} ; \vartheta\right)\right\}^{R_{i}}\right]\left[1-F_{X}(T ; \vartheta)\right]^{R_{J}^{*}}
$$

Where $B_{2}=\prod_{i=1}^{J}\left[n-\sum_{k=1}^{i-1}\left(1+R_{k}\right)\right]$

It is important to note that in our problem only partial information is available about the failure times of the items which is encoded with the help of fuzzy numbers. In light of the above let $\widetilde{x}_{1}, \widetilde{x}_{2}, \ldots, \widetilde{x}_{k}$ be the fuzzy lifetime of $\mathrm{k}$ failed items such that $\widetilde{x}_{i}=\left(a_{i}, b_{i}, c_{i}\right) ; i=1,2, \ldots, k$ with corresponding membership functions $\xi_{\widetilde{x}_{1}}(),. \xi_{\widetilde{x}_{2}}(),. \ldots, \xi_{\widetilde{x}_{k}}($.$) where$

$$
\xi_{\widetilde{x}_{i}}(x)=\left\{\begin{array}{cl}
\frac{x-\left(x_{i}-h_{i}\right)}{h_{i}} ; & x_{i}-h_{i} \leq x \leq x_{i} \\
\frac{\left(x_{i}+h_{i}\right)-x}{h_{i}} ; & x_{i} \leq x \leq x_{i}+h_{i} \\
0 ; & \text { otherwise }
\end{array}\right.
$$

Let $\widetilde{z}_{i 1}, \widetilde{z}_{i 2}, \ldots, \widetilde{z}_{i R_{i}} ; i=1,2, \ldots, k$ denotes the lifetime of those $R_{i}$ items which are removed from the experiment at the time of $i$ th failure and their membership function is defined as follows:

$$
\xi_{\widetilde{z}_{i j}}(z)= \begin{cases}0, & z \leq b_{i} \\ 1, & z>b_{i}\end{cases}
$$

As discussed above $T$ is maximum time for termination of the experiment and it is an exact number which is fixed before the experiment starts. It is important to mention here that an exact number can be treated as special case of fuzzy number for which MF takes only one value which is unity. Therefore, $T$ can be encoded as a fuzzy number $\widetilde{T}$ with membership function $\xi_{\widetilde{T}}(T)=1$ and mean of this fuzzy number will be $T$ itself. Hence lifetime of $R_{J}^{*}$ surviving items, which are removed from the test at prefixed time $T$ in case II, can be encoded as fuzzy numbers $\widetilde{y}_{1}, \widetilde{y}_{2}, \ldots, \widetilde{y}_{R_{J}^{*}}$ with the membership function

$$
\xi_{\widetilde{y}_{j}}(y)= \begin{cases}0, & y \leq T \\ 1, & y>T\end{cases}
$$

Thus the set of observed lifetimes in each case can be written as

Case I: $\widetilde{U}=\left(\widetilde{x}_{1}, \ldots, \widetilde{x}_{m}, \widetilde{z}_{1}, \ldots, \widetilde{z}_{m}\right)$

Case II: $\widetilde{U}=\left(\widetilde{x}_{1}, \ldots, \widetilde{x}_{J}, \widetilde{z}_{1}, \ldots, \widetilde{z}_{J}, \widetilde{y}_{T}\right)$

Where $\widetilde{U}$ is the observed fuzzy data and can be seen as an incomplete specification of the complete data vector $U, \widetilde{z}_{i}$ is a $1 \times R_{i}$ vector with $\widetilde{z}_{i}=\left(\widetilde{z}_{i 1}, \widetilde{z}_{i 2}, \ldots, \widetilde{z}_{i R_{i}}\right) ; i=1,2, \ldots, k$ and $\widetilde{y}_{T}$ is $1 \times R_{J}^{*}$ vector with $\widetilde{y}_{T}=\left(\widetilde{y}_{1}, \widetilde{y}_{2}, \ldots, \widetilde{y}_{R_{J}^{*}}\right)$. 


\section{Point estimation of parameter}

In this section, we will develop the methodology to obtain point estimates of population parameters using classical as well as Bayesian methods of estimation under Type-II progressive hybrid censoring using fuzzy data. In order to demonstrate the developed methodology we are assuming that the life times of the items put on test are independently and identically distributed as Rayleigh distribution with parameter $\vartheta$. The probability density function and the cumulative distribution function of Rayleigh distribution is given below:

$$
f(x)=2 \vartheta x e^{-\vartheta x^{2}} \quad ; x>0, \vartheta>0
$$

and

$$
F(x)=1-e^{-\vartheta x^{2}} \quad ; x>0, \vartheta>0
$$

where $\vartheta>0$ is a scale parameter.

\subsection{Estimation under classical setup}

Under classical paradigm, three methods of estimation are used to obtain the point estimate of parameter namely ML, MM and CE method of estimation. ML and MM methods are well known methods of estimation while the CA method is proposed to lower the computational efforts.

\section{Maximum likelihood (ML) estimation}

In this section we obtain the ML estimate of the parameter of the lifetime distribution using the observed fuzzy data vector $\widetilde{U}$. First we try to obtain the general form of likelihood equation in each of the above stated cases i.e. case I and case II. The joint membership function for the observed fuzzy data vector $\widetilde{U}$ in each of the two cases is written as follows:

Case I:

$$
\xi_{\widetilde{U}}(U)=\left(\prod_{i=1}^{m} \xi_{\widetilde{x}_{i}}(x)\right) \times\left(\prod_{i=1}^{m} \prod_{j=1}^{R_{i}} \xi_{\widetilde{z}_{i j}}(z)\right)
$$

Case II:

$$
\xi_{\widetilde{U}}(U)=\left(\prod_{i=1}^{J} \xi_{\widetilde{x}_{i}}(x)\right) \times\left(\prod_{i=1}^{J} \prod_{j=1}^{R_{i}} \xi_{\widetilde{z}_{i j}}(z)\right) \times\left(\prod_{j=1}^{R_{J}^{*}} \xi_{\widetilde{y}_{j}}(y)\right)
$$

The likelihood function for observed data $\widetilde{U}$ can be obtained by using Zadeh 's definition of the probability of a fuzzy event; see Zadeh (1968)

$$
l(\vartheta ; \widetilde{u})=P(\widetilde{u} ; \vartheta)=\int f(u ; \vartheta) \xi_{\widetilde{u}}(u) d u
$$

Using equations (5), (6) and (7), likelihood equation for the observed fuzzy data $\widetilde{U}$ for case I and II is given as:

$$
l(\vartheta ; \widetilde{u})=\left[\prod_{i=1}^{m} \int f(x ; \vartheta) \xi_{\widetilde{x}_{i}}(x) d x\right] \times\left[\prod_{i=1}^{m} \prod_{j=1}^{R_{i}} \int f(z ; \vartheta) \xi_{\widetilde{z}_{i j}}(z) d z\right]
$$

and

$$
l(\vartheta ; \widetilde{u})=\left[\prod_{i=1}^{J} \int f(x ; \vartheta) \xi_{\widetilde{x}_{i}}(x) d x\right] \times\left[\prod_{i=1}^{J} \prod_{j=1}^{R_{i}} \int f(z ; \vartheta) \xi_{\widetilde{z}_{i j}}(z) d z\right] \times\left[\prod_{j=1}^{R_{J}^{*}} \int f(y ; \vartheta) \xi_{\widetilde{y}_{j}}(y) d y\right]
$$


provided that $\mathrm{J} \geq 1$. It is important to note that MLE does not exists when the experiment is terminated at prefixed time $T$ before failure of any item.

Equation (8) and (9) can be used to write likelihood equation for Type-II progressively hybrid censored fuzzy data from a specified probability density function by simply substituting the form of density $f(x ; \vartheta)$ and membership function of fuzzy number $\xi_{\widetilde{x}}(x)$. Here we are assuming the form of density as given in equation (3) therefore log-likelihood for case I may be written as:

$$
\begin{aligned}
\log l(\vartheta ; \widetilde{u})=L(\vartheta ; \widetilde{u}) & =\sum_{i=1}^{m} \log \int 2 \vartheta x e^{-\vartheta x^{2}} \xi_{\widetilde{x}_{i}}(x) d x+\sum_{i=1}^{m} \sum_{j=1}^{R_{i}} \log \int 2 \vartheta z e^{-\vartheta z^{2}} \xi_{\widetilde{z}_{i j}}(z) d z \\
& =m \log \vartheta+\sum_{i=1}^{m} \log \int 2 x e^{-\vartheta x^{2}} \xi_{\widetilde{x}_{i}}(x) d x-\vartheta \sum_{i=1}^{m} R_{i} b_{(i)}^{2}
\end{aligned}
$$

Similarly, using equation (9), log-likelihood for case II may be written as

$$
\begin{array}{r}
\log l(\vartheta ; \widetilde{u})=L(\vartheta ; \widetilde{u})=\sum_{i=1}^{J} \log \int 2 \vartheta x e^{-\vartheta x^{2}} \xi_{\widetilde{x}_{i}}(x) d x+\sum_{i=1}^{J} \sum_{j=1}^{R_{i}} \log \int 2 \vartheta z e^{-\vartheta z^{2}} \xi_{\widetilde{z}_{i j}}(z) d z \\
+\sum_{j=1}^{R_{J}^{*}} \log \int 2 \vartheta y e^{-\vartheta y^{2}} \xi_{\widetilde{y}_{j}}(y) d y \\
L(\vartheta ; \widetilde{u})=J \log \vartheta+\sum_{i=1}^{J} \log \int 2 x e^{-\vartheta x^{2}} \xi_{\widetilde{x}_{i}}(x) d x-\vartheta\left[\sum_{i=1}^{J} R_{i} b_{(i)}^{2}+R_{J}^{*} T^{2}\right]
\end{array}
$$

The maximum likelihood estimator of the parameter $\vartheta$ can be obtained by differentiating log likelihood equations, given by equations (10) and (11), partially with respect to parameter $\vartheta$ and equating it to zero. The resulting equation is as follows:

$$
\frac{\partial L(\vartheta ; \widetilde{u})}{\partial \vartheta}=\frac{k}{\vartheta}-\sum_{i=1}^{k} \frac{\int x^{3} e^{-\vartheta x^{2}} \xi_{\widetilde{x}_{i}}(x) d x}{\int x e^{-\vartheta x^{2}} \xi_{\widetilde{x}_{i}}(x) d x}-A(i)=0
$$

Where $k=m$ and $A(i)=\sum_{i=1}^{m} R_{i} b_{(i)}^{2}$ for case I

and $k=J$ and $A(i)=\left[\sum_{i=1}^{J} R_{i} b_{(i)}^{2}+R_{J}^{*} T^{2}\right]$ for case II

Since equation (12) cannot be solved analytically therefore some existing numerical methods such as Newton-Raphson method, Expectation-Maximization (EM) algorithm or other iterative procedure may be used to obtain the ML estimate of the parameter. Here EM algorithm is employed to find the ML estimate of the parameter.

The Expectation-Maximization (EM) algorithm is a broadly applicable iterative approach for computation of maximum likelihood estimates. When the data is available in incomplete form other iterative procedures such as Newton-Raphson method, often, turn out to be more complicated and have low convergence rate on the other hand EM algorithm provides high convergence rate in such type of problems. In each iteration of the EM algorithm, there are two steps, called the Expectation step or the E-step and the Maximization step or the M-step. Due to this, the algorithm is called EM algorithm. Starting from suitable initial parameter values, the $\mathrm{E}$ and M-steps are repeated until convergence. As the log-likelihood is based partly on unobservable data, it is replaced by its conditional expectation given the observed data in the E-step. More precisely E-step uses the current fit for unknown parameter and observed data to find the complete-data log likelihood. In the M-step we calculate the parameter value by maximizing the expected log-likelihood function obtained in the E-step. For further details about EM algorithm see; McLachlan and Krishnan (2007), Dempster, Laird, 
and Rubin (1977). Here we are using the fuzzy EM algorithm proposed by Denœux (2011) to obtain the ML estimate of parameter because observed fuzzy data is incomplete specification of complete data.

The log-likelihood for the complete data can be written as:

$$
L_{c}(U, \vartheta)=n(\log 2+\log \vartheta)+\sum_{i=1}^{n} \log x_{i}-\vartheta D(i)
$$

Differentiating equation (13) with respect to $\vartheta$ and equating it to zero, the corresponding likelihood equation can be obtained as follows:

$$
\frac{n}{\vartheta}=D(i)
$$

Where $D(i)=\left[\sum_{i=1}^{m} x_{i}^{2}+\sum_{i=1}^{m} \sum_{j=1}^{R_{i}} z_{i j}^{2}\right]$ for Case I

and $D(i)=\left[\sum_{i=1}^{J} x_{i}^{2}+\sum_{i=1}^{J} \sum_{j=1}^{R_{i}} z_{i j}^{2}+\sum_{j=1}^{R_{J}^{*}} y_{j}^{2}\right]$ for Case II.

Therefore, employing the E-step in equation (14) it reduces in the following form at the $(h+1)^{t h}$ iteration for case I and case II respectively:

$$
\begin{gathered}
\frac{n}{\vartheta^{(h+1)}}=\left[\sum_{i=1}^{m} E\left(X_{i}^{2} \mid \widetilde{x}_{i} ; \vartheta^{(h)}\right)+\sum_{i=1}^{m} \sum_{j=1}^{R_{i}} E\left(Z_{i j}^{2} \mid \widetilde{z}_{i j} ; \vartheta^{(h)}\right)\right] \\
\frac{n}{\vartheta^{(h+1)}}=\left[\sum_{i=1}^{J} E\left(X_{i}^{2} \mid \widetilde{x}_{i} ; \vartheta^{(h)}\right)+\sum_{i=1}^{J} \sum_{j=1}^{R_{i}} E\left(Z_{i j}^{2} \mid \widetilde{z}_{i j} ; \vartheta^{(h)}\right)+\sum_{j=1}^{R_{J}^{*}} E\left(Y_{j}^{2} \mid \widetilde{u}_{j} ; \vartheta^{(h)}\right)\right]
\end{gathered}
$$

where

$$
E\left(W_{i}^{2} \mid \widetilde{w_{i}} ; \vartheta^{(h)}\right)=\frac{\int w^{3} e^{-\vartheta^{(h)} w^{2}} \xi_{\widetilde{w}_{i}}(w) d w}{\int w e^{-\vartheta^{(h)} w^{2}} \xi_{\widetilde{w}_{i}}(w) d w}
$$

In the M-step we have to maximize equations (15) or (16) to find the estimate of parameter after every iteration. Hence estimate of parameter in each of the two cases, at the $(h+1)^{t h}$ iteration, may be given respectively as:

$$
\begin{aligned}
& \hat{\vartheta}^{(h+1)}=n\left[\sum_{i=1}^{m} E\left(X_{i}^{2} \mid{\widetilde{x_{i}}}_{\vartheta^{(h)}}\right)+\sum_{i=1}^{m} \sum_{j=1}^{R_{i}} E\left(Z_{i j}^{2} \mid \widetilde{z}_{i j} ; \vartheta^{(h)}\right)\right]^{-1} \\
& \hat{\vartheta}^{(h+1)}=n\left[\sum_{i=1}^{J} E\left(X_{i}^{2} \mid \widetilde{x}_{i} ; \vartheta^{(h)}\right)+\sum_{i=1}^{J} \sum_{j=1}^{R_{i}} E\left(Z_{i j}^{2} \mid \widetilde{z}_{i j} ; \vartheta^{(h)}\right)+\sum_{j=1}^{R_{J}^{*}} E\left(Y_{j}^{2} \mid \widetilde{u}_{j} ; \vartheta^{(h)}\right)\right]^{-1}
\end{aligned}
$$

The iterative process is repeated until the convergence i.e. $\left|\hat{\vartheta}^{(h+1)}-\hat{\vartheta}^{(h)}\right|<\varepsilon$ for some $\varepsilon>0$. If the convergence occurs then the current $\hat{\vartheta}^{(h+1)}$ is the approximate maximum likelihood estimates of $\vartheta$ via EM algorithm.

\section{Method of conditional moments}

The method of moments is quite popular method of estimation in classical setup to find the estimates of unknown population parameters. In method of moments we equate different sample moments with corresponding population moments to find the parameter estimates. In this article we are concerned with the analysis of Type-II progressively hybrid censored data when the available data is imprecise and presented in form of fuzzy numbers. Therefore we 
can utilize method of conditional moments, for incomplete data, to obtain the moment estimate of population parameters which is recently proposed by Lu and Wang (2013). For this we have to equate the population moments with conditional expectation of sample moment given the observed data.

Let $X=\left(X_{1}, X_{1}, \ldots, X_{n}\right)$ be a random sample from density function $f(x, \theta)$ and $\mu_{k}(\theta)$ is the $k$ th non-central population moment. Whereas $m_{k}(X)$ is the $k$ th non-central sample moment. Let $Y$ be the incomplete observed sample then the moment estimators for $\theta=\left(\theta_{1}, \theta_{2}, \ldots, \theta_{p}\right)^{\prime}$ are the solution of following conditional moment equation:

$$
\mu_{k}(\theta)=E\left[m_{k}(X) \mid Y\right] \quad ; \quad k=1,2, \ldots, p
$$

In order to further explain the methodology we will obtain the conditional moment estimate for parameter of Rayleigh distribution. It is well known that $r$ th moment of Rayleigh distribution is given as:

$$
E\left(X^{r}\right)=\vartheta^{-\frac{r}{2}} \Gamma\left(\frac{r}{2}+1\right)
$$

We will use an iterative algorithm to find the parameter estimate. First we have to choose a suitable initial value of the unknown parameter and then sample moment is replaced by its conditional expectation given the observed data. Following equation, obtained by equating the first sample moment to the corresponding population moment, can be used to find the estimate of parameter using moment method in both cases

$$
\vartheta^{-\frac{1}{2}} \Gamma\left(\frac{1}{2}+1\right)=\frac{B(i)}{n}
$$

The solution for $\vartheta$, obtained by solving the above expressions, is as follows:

$$
\vartheta=\frac{n^{2} \pi}{4}\left[B(i)^{-2}\right]
$$

$$
\text { where } \quad \begin{aligned}
B(i) & =\left[\sum_{i=1}^{m} E\left(X_{i} \mid \widetilde{x}_{i}\right)+\sum_{i=1}^{m} \sum_{j=1}^{R_{i}} E\left(z_{i j} \mid \widetilde{z}_{i j}\right)\right] \text { for case I } \\
\text { and } & B(i)=\left[\sum_{i=1}^{J} E\left(X_{i} \mid \widetilde{x}_{i}\right)+\sum_{i=1}^{J} \sum_{j=1}^{R_{i}} E\left(z_{i j} \mid \widetilde{z}_{i j}\right)+\sum_{j=1}^{R_{J}^{*}} E\left(Y_{j} \mid \widetilde{y}_{j}\right)\right] \text { for case II }
\end{aligned}
$$

Parameter is updated at each iteration by its predicted value at the previous iteration. We will proceed in the same manner until the convergence occurs and current value of parameter at which convergence occur is the MM estimate of parameter. The estimated value of parameter at the $(h+1)$ th iteration

for case I:

$$
\vartheta^{(h+1)}=\frac{n^{2} \pi}{4}\left[\sum_{i=1}^{m} E\left(X \mid \widetilde{x}_{i} ; \vartheta^{(h)}\right)+\sum_{i=1}^{m} \sum_{j=1}^{R_{i}} E\left(Z \mid \widetilde{z}_{i j} ; \vartheta^{(h)}\right)\right]^{-2}
$$

and for case II:

$$
\vartheta^{(h+1)}=\frac{n^{2} \pi}{4}\left[\sum_{i=1}^{J} E\left(X \mid \widetilde{x}_{i} ; \vartheta^{(h)}\right)+\sum_{i=1}^{J} \sum_{j=1}^{R_{i}} E\left(Z \mid \widetilde{z}_{i j} ; \vartheta^{(h)}\right)+\sum_{j=1}^{R_{J}^{*}} E\left(Y \mid \widetilde{u}_{j} ; \vartheta^{(h)}\right)\right]^{-2}
$$


Where

$$
\begin{aligned}
& E\left(X \mid \widetilde{x_{i}} ; \vartheta^{(h)}\right)=\frac{\int x^{2} e^{-\vartheta^{(h)}} x^{2} \xi_{\widetilde{x}_{i}}(x) d x}{\int x e^{-\vartheta^{(h)} x^{2}} \xi_{\widetilde{x}_{i}}(x) d x} ; i=1,2, \ldots, m \\
& E\left(Z \mid \widetilde{z}_{i j} ; \vartheta^{(h)}\right)=\frac{\int z^{2} e^{-\vartheta^{(h)} z^{2}} \xi_{\widetilde{z}_{i j}}(z) d z}{\int z e^{-\vartheta^{(h)} z^{2}} \xi_{\widetilde{z}_{i j}}(z) d z} ; i=1,2, \ldots, m, j=1,2, \ldots, R_{i} \\
& E\left(Y \mid \widetilde{u_{j}} ; \vartheta^{(h)}\right)=\frac{\int y^{2} e^{-\vartheta^{(h)} y^{2}} \xi_{\widetilde{y}_{j}}(y) d y}{\int y e^{-\vartheta^{(h)} y^{2}} \xi_{\widetilde{y}_{j}}(y) d y} ; j=1,2, \ldots, R_{J}^{*}
\end{aligned}
$$

Estimated value of parameter $\vartheta$, at which convergence occurs, will provide us the method of moment (MM) estimate of population parameter.

\section{Computational approach estimation method}

In the present article we have modified some conventional methods of estimating lifetime parameters according to new situation where the observations are censored and available in form of fuzzy numbers. Although conventional estimation methods are vary efficient, they need a lots of computational efforts to estimate the population parameter. Therefore we will use a new approach to find the estimate of population parameter which is called computational approach estimation (CAE) method. In this method less computational efforts are required and mean squared errors are also reasonable. Recently, Pak and Chatrabgoun (2016) proposed CAE method of estimation to obtain a point estimate of exponential mean parameter under progressive Type-II censoring scheme, when the lifetime observations are fuzzy. Let $\widetilde{x}_{1}, \widetilde{x}_{2}, \ldots, \widetilde{x}_{k}$ be the fuzzy lifetime of $\mathrm{k}$ failed items, where $\widetilde{x}_{i}=\left(a_{i}, b_{i}, c_{i}\right) ; i=1,2, \ldots, k$. The mean fuzzy number, denoted by $\overline{\widetilde{x}}=(\bar{a}, \bar{b}, \bar{c})$, can be obtained as

$$
\bar{a}=\frac{\sum_{i=1}^{k} a_{i}}{k}, \bar{b}=\frac{\sum_{i=1}^{k} b_{i}}{k} \text { and } \bar{c}=\frac{\sum_{i=1}^{k} c_{i}}{k}
$$

Then the CAE estimate of parameter of Rayleigh distribution is obtained by replacing the fuzzy data in the likelihood equation with its de-fuzzified values. The CAE estimate of the parameter $\vartheta$ obtained in this way in case I and case II is given respectively by equations (22) and (23) as:

$$
\begin{aligned}
& \widetilde{\widetilde{\vartheta}}=\left[\widetilde{\widetilde{x}}^{2}+\frac{1}{m} \sum_{i=1}^{m} R_{i} b_{(i)}^{2}\right]^{-1} \\
& \widetilde{\widetilde{\vartheta}}=\left[\widetilde{\widetilde{x}}^{2}+\frac{1}{J}\left(\sum_{i=1}^{J} R_{i} b_{(i)}^{2}+R_{J}^{*} T^{2}\right)\right]^{-1}
\end{aligned}
$$

where

$$
\widetilde{\widetilde{x}}=\frac{\int x \xi_{\overline{\tilde{x}}}(x) d x}{\int \xi_{\overline{\widetilde{x}}}(x) d x}
$$

Here we are converting the mean fuzzy number $\overline{\widetilde{x}}$ into real number $\widetilde{\widetilde{x}}$, by using a method of de-fuzzification named as center of gravity de-fuzzification technique, to obtain the point estimate of the parameter $\vartheta$.

\subsection{Estimation under Bayesian setup}

In the recent decades Bayesian perspective is favored for statistical inferences because it helps to find useful prior information and utilize it in formulating better methods of estimation. In 
this section, we have developed the Bayesian estimation procedure and HPD interval for the parameter $\vartheta$ of Rayleigh distribution. Let us assume that $\vartheta$ has gamma prior with pdf

$$
\pi(\vartheta)=\frac{\beta^{\alpha}}{\Gamma(\alpha)} e^{-\beta \vartheta} \vartheta^{(\alpha-1)} ; \vartheta>0, \alpha>0, \beta>0
$$

Here, both the hyper parameters $\alpha$ and $\beta$ are assumed to be known. We compute the Bayes estimate of the unknown parameter under squared error loss function. Using the prior given in equation (24) and the likelihood functions given in equations (8) and (9), the posterior density of $\vartheta$ for the given data can be written as follows:

$$
\begin{aligned}
\pi(\vartheta \mid \widetilde{u}) & =\frac{l(\vartheta ; \widetilde{u}) \pi(\vartheta)}{\int_{0}^{\infty} l(\vartheta ; \widetilde{u}) \pi(\vartheta) d \vartheta} \\
& =\frac{J}{\int_{0}^{\infty} J d \vartheta}
\end{aligned}
$$

Where, for case I:

$$
J=e^{-\beta \vartheta} \vartheta^{\alpha-1} \times \prod_{i=1}^{m} \int 2 \vartheta x e^{-\vartheta x^{2}} \xi_{\widetilde{x}_{i}}(x) d x \times \prod_{i=1}^{m} \prod_{j=1}^{R_{i}} \int 2 \vartheta z e^{-\vartheta z^{2}} \xi_{\widetilde{z}_{i j}}(z) d z
$$

for case II:

$$
\begin{aligned}
J=e^{-\beta \vartheta} \vartheta^{\alpha-1} \times \prod_{i=1}^{J} \int 2 \vartheta x e^{-\vartheta x^{2}} \xi_{\widetilde{x}_{i}}(x) d x & \times \prod_{i=1}^{J} \prod_{j=1}^{R_{i}} \int 2 \vartheta z e^{-\vartheta z^{2}} \xi_{\widetilde{z}_{i j}}(z) d z \\
& \times \prod_{j=1}^{R_{J}^{*}} \int 2 \vartheta y e^{-\vartheta y^{2}} \xi_{\widetilde{y}_{j}}(y) d y
\end{aligned}
$$

Suppose $\mathrm{h}($.$) is a function of \vartheta$. Then, Bayes estimate of $\mathrm{h}($.$) under squared error loss function$ can be written as:

$$
\begin{aligned}
\hat{h}(\vartheta) & =E_{\pi}[h(\vartheta)] \\
& =\frac{\int_{0}^{\infty} h(\vartheta) J d \vartheta}{\int_{0}^{\infty} J d \vartheta}
\end{aligned}
$$

It may be noted from the equation (25) that there is no closed form for the estimator. Therefore, we use Markov Chain Monte Carlo (MCMC) method to simulate sample from posterior distribution in order to calculate the Bayes estimate of $\vartheta$ and also to construct its HPD interval. We observed that posterior density $\pi(\vartheta \mid \widetilde{u})$ is similar to normal distribution. Thus utilizing the concept of Metropolis-Hastings algorithm with normal proposal distribution, we can generate samples from the posterior density function; see Hastings (1970). Algorithm of simulation consists of following steps:

Step 1: Start with an initial guess value $\vartheta^{(0)}$

Step 2: Set $\mathrm{j}=1$ and generate a new candidate parameter value $\vartheta^{*}$ from proposal density $q\left(\vartheta^{(1)} \mid \vartheta^{(0)}\right)$.

Step 3: Accept candidate $\vartheta^{*}$ as

$$
\vartheta^{(j)}=\left\{\begin{array}{lll}
\vartheta^{*} & \text { with probability } & \rho\left(\vartheta^{*}, \vartheta^{(j-1)}\right) \\
\vartheta^{(j-1)} & \text { with probability } & 1-\rho\left(\vartheta^{*}, \vartheta^{(j-1)}\right)
\end{array}\right.
$$

where 


$$
\rho\left(\vartheta^{*}, \vartheta^{(j-1)}\right)=\min \left\{\frac{\pi\left(\vartheta^{*} \mid \widetilde{u}\right) q\left(\vartheta^{(j-1)} \mid \vartheta^{*}\right)}{\pi\left(\vartheta^{(j-1)} \mid \widetilde{u}\right) q\left(\vartheta^{*} \mid \vartheta^{(j-1)}\right)}, 1\right\}
$$

Step 4: Set $\mathrm{j}=\mathrm{j}+1$

Step 5: Repeat steps 2-4, M times and obtain $\vartheta^{(j)} ; \mathrm{j}=1,2, \ldots, \mathrm{M}$

Step 6: Obtain the Bayes estimates of the parameter $\vartheta$ under SELF as the mean of generated samples from the posterior densities i.e.

$$
E_{\pi}(\vartheta \mid \widetilde{u})=\frac{1}{M-M_{0}} \sum_{j=M_{0}+1}^{M} \vartheta^{(j)}
$$

Where $M_{0}$ is burn-in period.

Step 7: To construct the HPD credible intervals for $\vartheta$, order the simulated samples as $\vartheta^{(1)} \leq$ $\vartheta^{(2)} \leq \cdots \leq \vartheta^{(M)}$. Then construct all the $100(1-\alpha) \%$ credible intervals of $\vartheta$ as

$$
\left(\vartheta^{[1]}, \vartheta^{[M(1-\alpha)+1]}\right), \ldots,\left(\vartheta^{[M \alpha]}, \vartheta^{[M]}\right)
$$

Here $[\mathrm{X}]$ denotes the largest integer less than or equal to $\mathrm{X}$. Then the HPD credible interval of $\vartheta$ is that credible interval which has the shortest length.

\section{Simulation study}

In this section, we present some experimental results based on a Monte-Carlo simulation study to compare the performance of different methods based on Type-II progressive hybrid censored fuzzy lifetime data. Samples are generated from the considered model for the arbitrarily chosen value of the parameter. The comparison of the performance of the estimators has been made on the basis of mean squared error (MSE). For this purpose, first we have generated Type-II progressive hybrid censored lifetime data from the specified distribution using the algorithm given by Balakrishnan and Cramer (2014) for fixed value of parameter $n, m$ and censoring scheme. Each realization of $\mathrm{x}$ was then fuzzified with the corresponding membership function

$$
\xi_{\widetilde{x}_{i}}(x)=\left\{\begin{array}{cl}
\frac{x-\left(x_{i}-h_{i}\right)}{h_{i}} ; & x_{i}-h_{i} \leq x \leq x_{i} \\
\frac{\left(x_{i}+h_{i}\right)-x}{h_{i}} ; & x_{i} \leq x \leq x_{i}+h_{i} \\
0 ; & \text { otherwise }
\end{array}\right.
$$

where $h_{i}=0.05 x_{i}$. We are simulating the situation in which observer is unable to provide exact value of observation and an interval of plausible values $\left[x_{i}-h_{i}, x_{i}+h_{i}\right]$ is provided. Using these fuzzy numbers we obtained estimate of the unknown parameter $\vartheta$ using the four methods provided in the preceding section based on the generated sample. We have also obtained the average length of the HPD credible intervals. After that, we have repeated this process for sufficiently large number of times to obtain the average estimates of the parameter and to get the estimated MSEs of the estimators.

For this purpose, we have arbitrarily taken $\vartheta=1$. To observe the effect of variation in sample size $n$ and total number of prefixed failures $m$ on the estimated value of parameter $\vartheta$ we have taken $n=(20,30,40,50)$ and $m=(10,15,20,25)$. The various values of prefixed time of termination of experiment $T$ considered for the estimation of the parameter are 0.4, 0.6 and 0.8 . For further variation, we have considered six removal schemes for given values of $n$ and $m$ which are as follows:

Scheme 1: $R_{1}=R_{2}=\cdots=R_{m-1}=0$ and $R_{m}=n-m$;

Scheme 2: $R_{1}=n-m$ and $R_{2}=R_{3}=\cdots=R_{m}=0$; 
Scheme 3: $R_{1}=R_{2}=\cdots=R_{m-1}=1$ and $R_{m}=n-2 m+1$;

Scheme 4: $R_{1}=R_{2}=\cdots=R_{m-5}=0$ and $R_{m-4}=R_{m-3}=\cdots=R_{m}=\left(\frac{n-m}{5}\right)$;

Scheme 5: $R_{1}=\cdots=R_{(k-3)}=0=R_{(k+3)}=\cdots=R_{m}$ and $R_{k-2}=\cdots=R_{k+2}=\left(\frac{n-m}{5}\right)$

Where $\mathrm{k}$ is $\left(\frac{m}{2}\right)$ and $\left(\frac{m+1}{2}\right)$ respectively for even and odd value of $\mathrm{m}$;

Scheme 6: $R_{1}=R_{2}=\cdots=R_{5}=\left(\frac{n-m}{5}\right)$ and $R_{6}=R_{7}=\cdots=R_{m}=0$;

The samples of Type-II progressive hybrid censored fuzzy lifetime data were simulated using the procedure mentioned above. ML, MM and CA estimates of the parameter are calculated by taking suitable initial value of parameter in the iterative algorithms. Also, Bayes estimate under informative and non-informative priors along with their HPD intervals are calculated. For this purpose, we use non informative prior $\alpha=\beta=0$ but these priors are improper priors hence to make them proper prior we will take $\alpha=\beta=0.00001$. These results are same as those obtained for improper priors. In order to analyze the effect of informative prior on Bayes estimate we have taken three different sets of values for hyper parameters. For the choice of hyper parameters of informative prior, we have taken prior means equals to the true values of the parameter with varying prior variances. The prior variance indicates our confidence in the prior guess. A large prior variance shows less confidence in prior guess. On other hand, small prior variance indicates greater confidence in prior guess. In this study, we have taken three different values of prior variance viz. 0.5 (small), 10 (moderate) and 100 (large) and denoted it with prior 1, 2 and 3. We have generated posterior sample of size 30000 to obtain the Bayes estimate of parameter using the algorithm discussed in section 3.2 and then first 10000 MCMC iterations (Burn-in period) have been discarded from the generated sequence. We have repeated the whole process sufficient number times to obtain the average estimates, corresponding mean square errors (MSEs) and HPD interval corresponding to Bayes estimate. The results obtained in this way are summarized and presented in Tables 1-5.

From Tables 1-3 we note that as the sample size $(n)$, number of failures $(m)$ and prefixed time of termination $(T)$ increases MSE of all estimators decreases for fixed value of parameter. We may further note that MSE for scheme 1 is less than MSEs for all other schemes and MSE for scheme 2 is greater than MSEs for all other schemes. It may also be noted that the MSE of Bayes estimator is less than all other estimators followed by MLE, MME and CAE in most of the cases. From Table 4 we see that the average length of HPD interval decreases as sample size $(n)$, number of failures $(m)$ and prefixed time of termination $(T)$ increases for fix value of parameter and the coverage probability for HPD intervals is either equal to the prefixed value $(95 \%)$ or slightly less than that. From Table 5 we note that the for fixed value of $n$, $m, T$ and parameter $\vartheta$, MSE of Bayes estimator increases as prior variance increases when informative priors are used to obtain the estimate of parameter. We may further note that the average length of HPD interval increase as prior variance increases.

Table 1: Average MLE, MME, CAE and Bayes estimate of the parameter of Rayleigh distribution based on Type-II progressively hybrid censored fuzzy lifetime data along with corresponding MSE's for fixed value of parameter $\vartheta=1, T=0.4$ and varying $n, m$ and censoring scheme

\begin{tabular}{cccccccccc}
\hline $\mathbf{( n , m )}$ & Scheme & MLE & MSE & MME & MSE & CAE & MSE & Bayes & MSE \\
\hline$(20,10)$ & 1 & 1.0640 & 0.3322 & 1.0654 & 0.3406 & 1.0742 & 0.3478 & 1.0638 & 0.3320 \\
& 2 & 1.1981 & 0.6432 & 1.2150 & 0.6919 & 1.2142 & 0.6933 & 1.1976 & 0.6431 \\
& 3 & 1.0969 & 0.3843 & 1.0979 & 0.3984 & 1.1080 & 0.4095 & 1.0964 & 0.3842 \\
& 4 & 1.0633 & 0.3455 & 1.0644 & 0.3527 & 1.0735 & 0.3621 & 1.0631 & 0.3454 \\
& 5 & 1.1170 & 0.4235 & 1.1156 & 0.4309 & 1.1282 & 0.4458 & 1.1168 & 0.4234 \\
& 6 & 1.1436 & 0.4987 & 1.1445 & 0.5151 & 1.1559 & 0.5290 & 1.1436 & 0.4985 \\
\hline$(30,10)$ & 1 & 1.0278 & 0.2361 & 1.0277 & 0.2382 & 1.0375 & 0.2462 & 1.0274 & 0.2359 \\
& 2 & 1.1793 & 0.6266 & 1.2009 & 0.6814 & 1.1973 & 0.6810 & 1.1791 & 0.6264 \\
& 3 & 1.0479 & 0.2732 & 1.0493 & 0.2785 & 1.0584 & 0.2865 & 1.0477 & 0.2730 \\
\hline
\end{tabular}




\begin{tabular}{|c|c|c|c|c|c|c|c|c|c|}
\hline$(\mathbf{n}, \mathbf{m})$ & Scheme & MLE & MSE & MME & MSE & CAE & MSE & Bayes & MSE \\
\hline & 4 & 1.0314 & 0.2555 & 1.0299 & 0.2572 & 1.0410 & 0.2663 & 1.0311 & 0.2554 \\
\hline & 5 & 1.0869 & 0.3408 & 1.0834 & 0.3435 & 1.0976 & 0.3580 & 1.0862 & 0.3406 \\
\hline & 6 & 1.1163 & 0.4174 & 1.1150 & 0.4276 & 1.1286 & 0.4413 & 1.1160 & 0.4172 \\
\hline \multirow[t]{6}{*}{$(40,10)$} & 1 & 1.0154 & 0.1999 & 1.0155 & 0.2000 & 1.0249 & 0.2081 & 1.0150 & 0.1988 \\
\hline & 2 & 1.1721 & 0.6101 & 1.1969 & 0.6663 & 1.1916 & 0.6655 & 1.1720 & 0.6098 \\
\hline & 3 & 1.0257 & 0.2018 & 1.0248 & 0.2018 & 1.0356 & 0.2106 & 1.0253 & 0.2017 \\
\hline & 4 & 1.0476 & 0.2273 & 1.0458 & 0.2272 & 1.0575 & 0.2370 & 1.0475 & 0.2271 \\
\hline & 5 & 1.0992 & 0.3081 & 1.0950 & 0.3092 & 1.1100 & 0.3234 & 1.0990 & 0.3080 \\
\hline & 6 & 1.1462 & 0.4282 & 1.1428 & 0.4372 & 1.1591 & 0.4541 & 1.1458 & 0.4280 \\
\hline \multirow[t]{6}{*}{$(50,10)$} & 1 & 1.0410 & 0.1932 & 1.0370 & 0.1946 & 1.0507 & 0.2072 & 1.0368 & 0.1929 \\
\hline & 2 & 1.1519 & 0.5828 & 1.1804 & 0.6455 & 1.1716 & 0.6371 & 1.1516 & 0.5826 \\
\hline & 3 & 1.0370 & 0.1908 & 1.0343 & 0.1912 & 1.0470 & 0.2029 & 1.0370 & 0.1903 \\
\hline & 4 & 1.0626 & 0.2041 & 1.0582 & 0.2020 & 1.0724 & 0.2129 & 1.0622 & 0.2039 \\
\hline & 5 & 1.1218 & 0.3034 & 1.1156 & 0.3109 & 1.1330 & 0.3215 & 1.1209 & 0.3034 \\
\hline & 6 & 1.1514 & 0.3974 & 1.1472 & 0.4094 & 1.1641 & 0.4208 & 1.1511 & 0.3972 \\
\hline \multirow[t]{6}{*}{$(30,15)$} & 1 & 1.0280 & 0.2300 & 1.0286 & 0.2329 & 1.0378 & 0.2400 & 1.0270 & 0.2300 \\
\hline & 2 & 1.0908 & 0.4115 & 1.1004 & 0.4294 & 1.1045 & 0.4377 & 1.0903 & 0.4033 \\
\hline & 3 & 1.0480 & 0.2600 & 1.0488 & 0.2652 & 1.0583 & 0.2722 & 1.0479 & 0.2559 \\
\hline & 4 & 1.0282 & 0.2328 & 1.0290 & 0.2366 & 1.0379 & 0.2428 & 1.0280 & 0.2325 \\
\hline & 5 & 1.0504 & 0.2624 & 1.0496 & 0.2637 & 1.0605 & 0.2742 & 1.0504 & 0.2623 \\
\hline & 6 & 1.0887 & 0.3424 & 1.0901 & 0.3548 & 1.1001 & 0.3607 & 1.0884 & 0.3417 \\
\hline \multirow[t]{6}{*}{$(40,15)$} & 1 & 1.0168 & 0.1680 & 1.0178 & 0.1707 & 1.0263 & 0.1750 & 1.0161 & 0.1637 \\
\hline & 2 & 1.0910 & 0.4105 & 1.1026 & 0.4312 & 1.1061 & 0.4392 & 1.0902 & 0.4084 \\
\hline & 3 & 1.0298 & 0.1947 & 1.0306 & 0.1993 & 1.0398 & 0.2035 & 1.0294 & 0.1929 \\
\hline & 4 & 1.0167 & 0.1731 & 1.0170 & 0.1756 & 1.0262 & 0.1804 & 1.0167 & 0.1731 \\
\hline & 5 & 1.0450 & 0.2154 & 1.0432 & 0.2164 & 1.0549 & 0.2249 & 1.0446 & 0.2153 \\
\hline & 6 & 1.0987 & 0.3213 & 1.0979 & 0.3301 & 1.1104 & 0.3392 & 1.0983 & 0.3211 \\
\hline \multirow[t]{6}{*}{$(50,15)$} & 1 & 1.0111 & 0.1419 & 1.0113 & 0.1431 & 1.0205 & 0.1476 & 1.0103 & 0.1387 \\
\hline & 2 & 1.1024 & 0.4100 & 1.1173 & 0.4346 & 1.1189 & 0.4405 & 1.1020 & 0.4054 \\
\hline & 3 & 1.0254 & 0.1555 & 1.0262 & 0.1583 & 1.0352 & 0.1626 & 1.0251 & 0.1508 \\
\hline & 4 & 1.0156 & 0.1454 & 1.0161 & 0.1469 & 1.0252 & 0.1515 & 1.0153 & 0.1454 \\
\hline & 5 & 1.0570 & 0.1954 & 1.0533 & 0.1938 & 1.0668 & 0.2038 & 1.0564 & 0.1953 \\
\hline & 6 & 1.1177 & 0.3030 & 1.1182 & 0.3162 & 1.1300 & 0.3206 & 1.1174 & 0.3030 \\
\hline \multirow[t]{6}{*}{$(40,20)$} & 1 & 1.0138 & 0.1766 & 1.0143 & 0.1786 & 1.0233 & 0.1839 & 1.0135 & 0.1765 \\
\hline & 2 & 1.0582 & 0.3092 & 1.0659 & 0.3222 & 1.0709 & 0.3269 & 1.0575 & 0.3089 \\
\hline & 3 & 1.0296 & 0.1958 & 1.0307 & 0.1987 & 1.0397 & 0.2047 & 1.0296 & 0.1957 \\
\hline & 4 & 1.0281 & 0.1792 & 1.0267 & 0.1804 & 1.0379 & 0.1871 & 1.0281 & 0.1790 \\
\hline & 5 & 1.0249 & 0.1914 & 1.0239 & 0.1923 & 1.0345 & 0.1994 & 1.0246 & 0.1913 \\
\hline & 6 & 1.0754 & 0.2640 & 1.0769 & 0.2737 & 1.0867 & 0.2781 & 1.0750 & 0.2638 \\
\hline \multirow[t]{6}{*}{$(50,20)$} & 1 & 1.0142 & 0.1370 & 1.0152 & 0.1392 & 1.0236 & 0.1427 & 1.0141 & 0.1369 \\
\hline & 2 & 1.0580 & 0.3048 & 1.0661 & 0.3146 & 1.0715 & 0.3233 & 1.0572 & 0.3046 \\
\hline & 3 & 1.0239 & 0.1556 & 1.0243 & 0.1583 & 1.0337 & 0.1624 & 1.0231 & 0.1554 \\
\hline & 4 & 1.0103 & 0.1373 & 1.0106 & 0.1389 & 1.0197 & 0.1428 & 1.0100 & 0.1372 \\
\hline & 5 & 1.0265 & 0.1648 & 1.0253 & 0.1650 & 1.0361 & 0.1715 & 1.0263 & 0.1647 \\
\hline & 6 & 1.0854 & 0.2475 & 1.0864 & 0.2569 & 1.0971 & 0.2617 & 1.0852 & 0.2474 \\
\hline \multirow[t]{6}{*}{$(50,25)$} & 1 & 1.0107 & 0.1399 & 1.0112 & 0.1417 & 1.0201 & 0.1456 & 1.0106 & 0.1397 \\
\hline & 2 & 1.0432 & 0.2553 & 1.0483 & 0.2613 & 1.0557 & 0.2696 & 1.0430 & 0.2552 \\
\hline & 3 & 1.0259 & 0.1540 & 1.0258 & 0.1553 & 1.0356 & 0.1607 & 1.0253 & 0.1538 \\
\hline & 4 & 1.0069 & 0.1357 & 1.0069 & 0.1371 & 1.0161 & 0.1410 & 1.0061 & 0.1356 \\
\hline & 5 & 1.0176 & 0.1455 & 1.0174 & 0.1463 & 1.0272 & 0.1516 & 1.0173 & 0.1454 \\
\hline & 6 & 1.0663 & 0.2184 & 1.0688 & 0.2277 & 1.0776 & 0.2304 & 1.0660 & 0.2180 \\
\hline
\end{tabular}


Table 2: Average MLE, MME, CAE and Bayes estimate of the parameter of Rayleigh distribution based on Type-II progressively hybrid censored fuzzy lifetime data along with corresponding MSE's for fixed value of parameter $\vartheta=1, T=0.6$ and varying $n, m$ and censoring scheme

\begin{tabular}{|c|c|c|c|c|c|c|c|c|c|}
\hline$(\mathbf{n}, \mathbf{m})$ & Scheme & MLE & MSE & MME & MSE & CAE & MSE & Bayes & MSE \\
\hline \multirow[t]{6}{*}{$(20,10)$} & 1 & 1.0395 & 0.2011 & 1.0428 & 0.2059 & 1.0627 & 0.2229 & 1.0394 & 0.2010 \\
\hline & 2 & 1.0964 & 0.3605 & 1.1071 & 0.3886 & 1.1279 & 0.4147 & 1.0961 & 0.3601 \\
\hline & 3 & 1.0638 & 0.2440 & 1.0629 & 0.2507 & 1.0884 & 0.2721 & 1.0633 & 0.2437 \\
\hline & 4 & 1.0616 & 0.2259 & 1.0583 & 0.2266 & 1.0846 & 0.2485 & 1.0580 & 0.2243 \\
\hline & 5 & 1.0784 & 0.2631 & 1.0776 & 0.2687 & 1.1025 & 0.2923 & 1.0781 & 0.2630 \\
\hline & 6 & 1.1123 & 0.3163 & 1.1123 & 0.3347 & 1.1398 & 0.3612 & 1.1120 & 0.3161 \\
\hline \multirow[t]{6}{*}{$(30,10)$} & 1 & 1.0750 & 0.2006 & 1.0664 & 0.2035 & 1.0967 & 0.2161 & 1.0662 & 0.2003 \\
\hline & 2 & 1.0944 & 0.3549 & 1.1086 & 0.3843 & 1.1305 & 0.4153 & 1.0938 & 0.3545 \\
\hline & 3 & 1.0402 & 0.1828 & 1.0388 & 0.1832 & 1.0637 & 0.2015 & 1.0400 & 0.1828 \\
\hline & 4 & 1.0836 & 0.1932 & 1.0768 & 0.1915 & 1.1060 & 0.2126 & 1.0766 & 0.1914 \\
\hline & 5 & 1.1157 & 0.2500 & 1.1117 & 0.2572 & 1.1405 & 0.2777 & 1.1153 & 0.2497 \\
\hline & 6 & 1.1073 & 0.2642 & 1.1028 & 0.2699 & 1.1349 & 0.3013 & 1.1070 & 0.2641 \\
\hline \multirow[t]{6}{*}{$(40,10)$} & 1 & 1.1024 & 0.1661 & 1.0893 & 0.1726 & 1.1204 & 0.1827 & 1.0890 & 0.1661 \\
\hline & 2 & 1.1077 & 0.3585 & 1.1256 & 0.3847 & 1.1482 & 0.4259 & 1.1074 & 0.3581 \\
\hline & 3 & 1.0910 & 0.1599 & 1.0789 & 0.1662 & 1.1118 & 0.1787 & 1.0786 & 0.1597 \\
\hline & 4 & 1.0780 & 0.1648 & 1.0670 & 0.1697 & 1.0986 & 0.1844 & 1.0664 & 0.1647 \\
\hline & 5 & 1.1191 & 0.2214 & 1.1083 & 0.2228 & 1.1429 & 0.2523 & 1.1080 & 0.2210 \\
\hline & 6 & 1.1330 & 0.2673 & 1.1334 & 0.2840 & 1.1621 & 0.3087 & 1.1329 & 0.2672 \\
\hline \multirow[t]{6}{*}{$(50,10)$} & 1 & 1.0943 & 0.1593 & 1.1000 & 0.1644 & 1.1083 & 0.1860 & 1.0942 & 0.1593 \\
\hline & 2 & 1.0870 & 0.3439 & 1.1107 & 0.3766 & 1.1264 & 0.4120 & 1.0862 & 0.3438 \\
\hline & 3 & 1.1040 & 0.1663 & 1.0909 & 0.1688 & 1.1204 & 0.1951 & 1.1040 & 0.1663 \\
\hline & 4 & 1.0882 & 0.1635 & 1.0778 & 0.1689 & 1.1069 & 0.1805 & 1.0771 & 0.1634 \\
\hline & 5 & 1.1394 & 0.2331 & 1.1331 & 0.2415 & 1.1634 & 0.2629 & 1.1390 & 0.2330 \\
\hline & 6 & 1.1275 & 0.2587 & 1.1278 & 0.2768 & 1.1565 & 0.2994 & 1.1271 & 0.2525 \\
\hline \multirow[t]{6}{*}{$(30,15)$} & 1 & 1.0187 & 0.1191 & 1.0178 & 0.1203 & 1.0406 & 0.1308 & 1.0184 & 0.1190 \\
\hline & 2 & 1.0508 & 0.2275 & 1.0561 & 0.2349 & 1.0801 & 0.2583 & 1.0502 & 0.2273 \\
\hline & 3 & 1.0389 & 0.1474 & 1.0394 & 0.1512 & 1.0623 & 0.1642 & 1.0382 & 0.1472 \\
\hline & 4 & 1.0192 & 0.1233 & 1.0186 & 0.1249 & 1.0415 & 0.1360 & 1.0190 & 0.1233 \\
\hline & 5 & 1.0606 & 0.1620 & 1.0566 & 0.1640 & 1.0833 & 0.1796 & 1.0618 & 0.1617 \\
\hline & 6 & 1.0669 & 0.1998 & 1.0675 & 0.2086 & 1.0935 & 0.2286 & 1.0665 & 0.1996 \\
\hline \multirow[t]{6}{*}{$(40,15)$} & 1 & 1.0284 & 0.1050 & 1.0257 & 0.1066 & 1.0502 & 0.1166 & 1.0251 & 0.1048 \\
\hline & 2 & 1.0628 & 0.2291 & 1.0704 & 0.2394 & 1.0945 & 0.2635 & 1.0623 & 0.2290 \\
\hline & 3 & 1.0359 & 0.1157 & 1.0350 & 0.1176 & 1.0593 & 0.1293 & 1.0353 & 0.1156 \\
\hline & 4 & 1.0360 & 0.1064 & 1.0327 & 0.1060 & 1.0575 & 0.1172 & 1.0320 & 0.1060 \\
\hline & 5 & 1.0686 & 0.1421 & 1.0630 & 0.1434 & 1.0903 & 0.1569 & 1.0683 & 0.1420 \\
\hline & 6 & 1.0821 & 0.1882 & 1.0858 & 0.2005 & 1.1101 & 0.2168 & 1.0820 & 0.1880 \\
\hline \multirow[t]{6}{*}{$(50,15)$} & 1 & 1.0442 & 0.1002 & 1.0377 & 0.0969 & 1.0642 & 0.1077 & 1.0374 & 0.0968 \\
\hline & 2 & 1.0557 & 0.2268 & 1.0657 & 0.2391 & 1.0890 & 0.2621 & 1.0555 & 0.2264 \\
\hline & 3 & 1.0433 & 0.1053 & 1.0398 & 0.1070 & 1.0660 & 0.1177 & 1.0394 & 0.1053 \\
\hline & 4 & 1.0475 & 0.1004 & 1.0416 & 0.1020 & 1.0678 & 0.1110 & 1.0413 & 0.1004 \\
\hline & 5 & 1.0823 & 0.1382 & 1.0764 & 0.1406 & 1.1034 & 0.1531 & 1.0820 & 0.1380 \\
\hline & 6 & 1.0790 & 0.1772 & 1.0855 & 0.1919 & 1.1078 & 0.2063 & 1.0783 & 0.1769 \\
\hline \multirow[t]{4}{*}{$(40,20)$} & 1 & 1.0178 & 0.0881 & 1.0191 & 0.0901 & 1.0400 & 0.0974 & 1.0171 & 0.0880 \\
\hline & 2 & 1.0416 & 0.1730 & 1.0477 & 0.1797 & 1.0695 & 0.1956 & 1.0415 & 0.1730 \\
\hline & 3 & 1.0342 & 0.1092 & 1.0345 & 0.1122 & 1.0576 & 0.1221 & 1.0340 & 0.1090 \\
\hline & 4 & 1.0173 & 0.0903 & 1.0176 & 0.0915 & 1.0393 & 0.0997 & 1.0169 & 0.0900 \\
\hline
\end{tabular}




\begin{tabular}{cccccccccc}
\hline $\mathbf{( n , m )}$ & Scheme & MLE & MSE & MME & MSE & CAE & MSE & Bayes & MSE \\
\hline & 5 & 1.0514 & 0.1185 & 1.0475 & 0.1199 & 1.0732 & 0.1311 & 1.0513 & 0.1184 \\
& 6 & 1.0490 & 0.1497 & 1.0532 & 0.1583 & 1.0756 & 0.1709 & 1.0482 & 0.1494 \\
\hline$(50,20)$ & 1 & 1.0194 & 0.0761 & 1.0179 & 0.0764 & 1.0410 & 0.0841 & 1.0174 & 0.0760 \\
& 2 & 1.0417 & 0.1705 & 1.0474 & 0.1775 & 1.0709 & 0.1934 & 1.0413 & 0.1703 \\
& 3 & 1.0281 & 0.0862 & 1.0283 & 0.0884 & 1.0511 & 0.0964 & 1.0280 & 0.0862 \\
& 4 & 1.0209 & 0.0795 & 1.0197 & 0.0794 & 1.0425 & 0.0874 & 1.0209 & 0.0793 \\
& 5 & 1.0569 & 0.1058 & 1.0519 & 0.1066 & 1.0777 & 0.1167 & 1.0566 & 0.1056 \\
& 6 & 1.0560 & 0.1440 & 1.0608 & 0.1540 & 1.0842 & 0.1665 & 1.0558 & 0.1436 \\
\hline$(50,25)$ & 1 & 1.0076 & 0.0679 & 1.0079 & 0.0694 & 1.0291 & 0.0749 & 1.0075 & 0.0679 \\
& 2 & 1.0304 & 0.1364 & 1.0333 & 0.1404 & 1.0572 & 0.1536 & 1.0300 & 0.1362 \\
& 3 & 1.0273 & 0.0857 & 1.0276 & 0.0878 & 1.0502 & 0.0956 & 1.0271 & 0.0856 \\
& 4 & 1.0120 & 0.0709 & 1.0119 & 0.0722 & 1.0338 & 0.0783 & 1.0120 & 0.0708 \\
& 5 & 1.0380 & 0.0883 & 1.0353 & 0.0890 & 1.0590 & 0.0971 & 1.0378 & 0.0883 \\
& 6 & 1.0449 & 0.1208 & 1.0486 & 0.1254 & 1.0720 & 0.1381 & 1.0443 & 0.1207 \\
\hline
\end{tabular}

Table 3: Average MLE, MME, CAE and Bayes estimate of the parameter of Rayleigh distribution based on Type-II progressively hybrid censored fuzzy lifetime data along with corresponding MSE's for fixed value of parameter $\vartheta=1, T=0.8$ and varying $n, m$ and censoring scheme

\begin{tabular}{cccccccccc}
\hline (n,m) & Scheme & MLE & MSE & MME & MSE & CAE & MSE & Bayes & MSE \\
\hline$(20,10)$ & 1 & 1.0857 & 0.1688 & 1.0748 & 0.1776 & 1.1251 & 0.2052 & 1.0855 & 0.1686 \\
& 2 & 1.0668 & 0.2588 & 1.0729 & 0.2740 & 1.1215 & 0.3301 & 1.0665 & 0.2584 \\
& 3 & 1.0789 & 0.2030 & 1.0762 & 0.2057 & 1.1253 & 0.2499 & 1.0789 & 0.2027 \\
& 4 & 1.0846 & 0.1798 & 1.0767 & 0.1807 & 1.1258 & 0.2138 & 1.0841 & 0.1798 \\
& 5 & 1.1104 & 0.2159 & 1.1046 & 0.2276 & 1.1552 & 0.2679 & 1.1100 & 0.2155 \\
& 6 & 1.0880 & 0.2276 & 1.0912 & 0.2450 & 1.1374 & 0.2886 & 1.0876 & 0.2271 \\
\hline$(30,10)$ & 1 & 1.0999 & 0.1687 & 1.0823 & 0.1729 & 1.1258 & 0.1872 & 1.0820 & 0.1683 \\
& 2 & 1.0644 & 0.2508 & 1.0764 & 0.2758 & 1.1254 & 0.3367 & 1.0642 & 0.2500 \\
& 3 & 1.0935 & 0.1653 & 1.0803 & 0.1730 & 1.1283 & 0.1938 & 1.0800 & 0.1649 \\
& 4 & 1.0935 & 0.1771 & 1.0827 & 0.1800 & 1.1282 & 0.2053 & 1.0813 & 0.1769 \\
& 5 & 1.1137 & 0.2183 & 1.1069 & 0.2220 & 1.1575 & 0.2746 & 1.1135 & 0.2179 \\
& 6 & 1.1017 & 0.2228 & 1.1067 & 0.2437 & 1.1569 & 0.2969 & 1.1014 & 0.2221 \\
\hline$(40,10)$ & 1 & 1.1154 & 0.1658 & 1.1003 & 0.1703 & 1.1340 & 0.1803 & 1.1002 & 0.1658 \\
& 2 & 1.0670 & 0.2520 & 1.0775 & 0.2735 & 1.1319 & 0.3366 & 1.0669 & 0.2509 \\
& 3 & 1.1046 & 0.1589 & 1.0896 & 0.1638 & 1.1277 & 0.1759 & 1.0895 & 0.1582 \\
& 4 & 1.1089 & 0.1647 & 1.0946 & 0.1693 & 1.1367 & 0.1867 & 1.0946 & 0.1644 \\
& 5 & 1.1180 & 0.1953 & 1.1118 & 0.2007 & 1.1601 & 0.2412 & 1.1180 & 0.1952 \\
& 6 & 1.0868 & 0.2063 & 1.0891 & 0.2182 & 1.1412 & 0.2722 & 1.0864 & 0.2059 \\
\hline$(50,10)$ & 1 & 1.1293 & 0.1650 & 1.1156 & 0.1697 & 1.1442 & 0.1779 & 1.1156 & 0.1642 \\
& 2 & 1.0915 & 0.2692 & 1.1019 & 0.2890 & 1.1630 & 0.3511 & 1.0914 & 0.2689 \\
& 3 & 1.1112 & 0.1575 & 1.0977 & 0.1625 & 1.1280 & 0.1709 & 1.0977 & 0.1572 \\
& 4 & 1.1105 & 0.1743 & 1.0969 & 0.1779 & 1.1334 & 0.1913 & 1.0968 & 0.1740 \\
& 5 & 1.1133 & 0.2009 & 1.1118 & 0.2121 & 1.1554 & 0.2515 & 1.1131 & 0.2004 \\
& 6 & 1.1129 & 0.2137 & 1.1180 & 0.2326 & 1.1702 & 0.2887 & 1.1126 & 0.2132 \\
\hline$(30,15)$ & 1 & 1.0486 & 0.0977 & 1.0394 & 0.1023 & 1.0868 & 0.1188 & 1.0392 & 0.0975 \\
& 2 & 1.0550 & 0.1637 & 1.0597 & 0.1732 & 1.1076 & 0.2092 & 1.0548 & 0.1636 \\
& 3 & 1.0463 & 0.1047 & 1.0424 & 0.1062 & 1.0888 & 0.1279 & 1.0462 & 0.1046 \\
& 4 & 1.0684 & 0.1064 & 1.0602 & 0.1088 & 1.1077 & 0.1298 & 1.0601 & 0.1063 \\
& 5 & 1.0617 & 0.1156 & 1.0559 & 0.1199 & 1.1009 & 0.1408 & 1.0617 & 0.1154 \\
\hline
\end{tabular}




\begin{tabular}{|c|c|c|c|c|c|c|c|c|c|}
\hline$(\mathbf{n}, \mathbf{m})$ & Scheme & MLE & MSE & MME & MSE & CAE & MSE & Bayes & MSE \\
\hline & 6 & 1.0594 & 0.1428 & 1.0656 & 0.1527 & 1.1110 & 0.1845 & 1.0590 & 0.1425 \\
\hline \multirow[t]{6}{*}{$(40,15)$} & 1 & 1.0681 & 0.0941 & 1.0575 & 0.0965 & 1.0980 & 0.1068 & 1.0575 & 0.0940 \\
\hline & 2 & 1.0440 & 0.1611 & 1.0485 & 0.1689 & 1.0970 & 0.2030 & 1.0436 & 0.1610 \\
\hline & 3 & 1.0521 & 0.0967 & 1.0443 & 0.0997 & 1.0908 & 0.1164 & 1.0442 & 0.0966 \\
\hline & 4 & 1.0666 & 0.0995 & 1.0599 & 0.0992 & 1.1002 & 0.1140 & 1.0598 & 0.0991 \\
\hline & 5 & 1.0775 & 0.1170 & 1.0735 & 0.1205 & 1.1159 & 0.1431 & 1.0773 & 0.1169 \\
\hline & 6 & 1.0676 & 0.1414 & 1.0726 & 0.1509 & 1.1219 & 0.1871 & 1.0676 & 0.1411 \\
\hline \multirow[t]{6}{*}{$(50,15)$} & 1 & 1.0825 & 0.0899 & 1.0711 & 0.0924 & 1.1051 & 0.1002 & 1.0711 & 0.0892 \\
\hline & 2 & 1.0517 & 0.1569 & 1.0603 & 0.1695 & 1.1077 & 0.2020 & 1.0512 & 0.1565 \\
\hline & 3 & 1.0545 & 0.0869 & 1.0453 & 0.0876 & 1.0834 & 0.0968 & 1.0545 & 0.0865 \\
\hline & 4 & 1.0684 & 0.0944 & 1.0575 & 0.0971 & 1.0956 & 0.1073 & 1.0575 & 0.0942 \\
\hline & 5 & 1.0679 & 0.1169 & 1.0622 & 0.1219 & 1.1049 & 0.1441 & 1.0677 & 0.1164 \\
\hline & 6 & 1.0740 & 0.1409 & 1.0778 & 0.1536 & 1.1315 & 0.1892 & 1.0740 & 0.1405 \\
\hline \multirow[t]{6}{*}{$(40,20)$} & 1 & 1.0351 & 0.0705 & 1.0292 & 0.0722 & 1.0733 & 0.0850 & 1.0351 & 0.0725 \\
\hline & 2 & 1.0372 & 0.1169 & 1.0411 & 0.1240 & 1.0861 & 0.1465 & 1.0371 & 0.1167 \\
\hline & 3 & 1.0329 & 0.0754 & 1.0329 & 0.0787 & 1.0749 & 0.0932 & 1.0327 & 0.0753 \\
\hline & 4 & 1.0392 & 0.0720 & 1.0352 & 0.0716 & 1.0770 & 0.0853 & 1.0392 & 0.0716 \\
\hline & 5 & 1.0507 & 0.0835 & 1.0498 & 0.0879 & 1.0884 & 0.1031 & 1.0504 & 0.0831 \\
\hline & 6 & 1.0305 & 0.1006 & 1.0338 & 0.1070 & 1.0801 & 0.1289 & 1.0305 & 0.0906 \\
\hline \multirow[t]{6}{*}{$(50,20)$} & 1 & 1.0493 & 0.0643 & 1.0410 & 0.0660 & 1.0809 & 0.0745 & 1.0493 & 0.0640 \\
\hline & 2 & 1.0375 & 0.1225 & 1.0391 & 0.1296 & 1.0883 & 0.1543 & 1.0372 & 0.1224 \\
\hline & 3 & 1.0338 & 0.0745 & 1.0305 & 0.0747 & 1.0743 & 0.0896 & 1.0338 & 0.0741 \\
\hline & 4 & 1.0372 & 0.0673 & 1.0314 & 0.0676 & 1.0701 & 0.0773 & 1.0372 & 0.0671 \\
\hline & 5 & 1.0527 & 0.0880 & 1.0482 & 0.0913 & 1.0911 & 0.1097 & 1.0526 & 0.0879 \\
\hline & 6 & 1.0389 & 0.1054 & 1.0458 & 0.1120 & 1.0911 & 0.1361 & 1.0387 & 0.1053 \\
\hline \multirow[t]{6}{*}{$(50,25)$} & 1 & 1.0265 & 0.0507 & 1.0227 & 0.0518 & 1.0648 & 0.0616 & 1.0265 & 0.0504 \\
\hline & 2 & 1.0382 & 0.0966 & 1.0406 & 0.1026 & 1.0862 & 0.1221 & 1.0380 & 0.0962 \\
\hline & 3 & 1.0275 & 0.0597 & 1.0261 & 0.0623 & 1.0687 & 0.0740 & 1.0275 & 0.0596 \\
\hline & 4 & 1.0271 & 0.0542 & 1.0238 & 0.0547 & 1.0648 & 0.0646 & 1.0270 & 0.0538 \\
\hline & 5 & 1.0380 & 0.0637 & 1.0360 & 0.0676 & 1.0745 & 0.0778 & 1.0380 & 0.0636 \\
\hline & 6 & 1.0175 & 0.0821 & 1.0218 & 0.0863 & 1.0661 & 0.1041 & 1.0175 & 0.0811 \\
\hline
\end{tabular}

Table 4: Lower limit, upper limit, width and corresponding coverage probabilities of HPD credible interval for fixed value of parameter $\vartheta=1$ with varying time of termination i.e. $T=0.4,0.6,0.8$

\begin{tabular}{|c|c|c|c|c|c|c|c|c|c|c|c|c|c|}
\hline \multirow{2}{*}{$(\mathbf{n}, \mathbf{m})$} & \multirow{2}{*}{ Scheme } & \multicolumn{4}{|c|}{$\mathrm{T}=0.4$} & \multicolumn{4}{|c|}{$\mathbf{T}=0.6$} & \multicolumn{4}{|c|}{$\mathbf{T}=0.8$} \\
\hline & & LL & UL & HPD & CP & LL & UL & HPD & CP & LL & UL & HPD & CP \\
\hline \multirow[t]{6}{*}{$(20,10)$} & 1 & 0.17 & 2.20 & 2.03 & 0.83 & 0.34 & 1.88 & 1.54 & 0.89 & 0.44 & 1.80 & 1.36 & 0.94 \\
\hline & 2 & 0.09 & 2.76 & 2.67 & 0.97 & 0.20 & 2.25 & 2.05 & 0.90 & 0.28 & 1.97 & 1.69 & 0.93 \\
\hline & 3 & 0.16 & 2.35 & 2.19 & 0.89 & 0.29 & 1.94 & 1.65 & 0.93 & 0.39 & 1.86 & 1.47 & 0.94 \\
\hline & 4 & 0.18 & 2.25 & 2.07 & 0.84 & 0.33 & 1.89 & 1.56 & 0.88 & 0.42 & 1.83 & 1.40 & 0.93 \\
\hline & 5 & 0.17 & 2.32 & 2.15 & 0.86 & 0.31 & 2.02 & 1.72 & 0.91 & 0.37 & 1.90 & 1.53 & 0.94 \\
\hline & 6 & 0.16 & 2.53 & 2.37 & 0.92 & 0.27 & 2.14 & 1.87 & 0.92 & 0.33 & 1.94 & 1.61 & 0.93 \\
\hline \multirow[t]{6}{*}{$(30,10)$} & 1 & 0.25 & 1.96 & 1.70 & 0.93 & 0.44 & 1.83 & 1.39 & 0.92 & 0.48 & 1.80 & 1.32 & 0.94 \\
\hline & 2 & 0.09 & 2.63 & 2.54 & 0.92 & 0.19 & 2.14 & 1.95 & 0.91 & 0.29 & 1.97 & 1.69 & 0.93 \\
\hline & 3 & 0.24 & 2.02 & 1.78 & 0.95 & 0.40 & 1.82 & 1.41 & 0.93 & 0.39 & 1.86 & 1.47 & 0.94 \\
\hline & 4 & 0.26 & 2.00 & 1.74 & 0.94 & 0.41 & 1.84 & 1.43 & 0.91 & 0.46 & 1.80 & 1.34 & 0.95 \\
\hline & 5 & 0.24 & 2.14 & 1.90 & 0.94 & 0.36 & 1.96 & 1.61 & 0.93 & 0.40 & 1.87 & 1.47 & 0.93 \\
\hline & 6 & 0.19 & 2.27 & 2.08 & 0.93 & 0.31 & 2.11 & 1.80 & 0.91 & 0.36 & 1.95 & 1.59 & 0.94 \\
\hline \multirow[t]{5}{*}{$(40,10)$} & 1 & 0.32 & 1.83 & 1.51 & 0.94 & 0.48 & 1.83 & 1.35 & 0.94 & 0.48 & 1.81 & 1.33 & 0.96 \\
\hline & 2 & 0.10 & 2.64 & 2.54 & 0.92 & 0.21 & 2.20 & 2.00 & 0.90 & 0.30 & 2.01 & 1.71 & 0.93 \\
\hline & 3 & 0.31 & 1.89 & 1.58 & 0.92 & 0.45 & 1.77 & 1.33 & 0.92 & 0.47 & 1.77 & 1.30 & 0.95 \\
\hline & 4 & 0.31 & 1.87 & 1.56 & 0.93 & 0.46 & 1.83 & 1.37 & 0.95 & 0.47 & 1.81 & 1.33 & 0.94 \\
\hline & 5 & 0.29 & 2.06 & 1.77 & 0.93 & 0.38 & 1.94 & 1.55 & 0.93 & 0.42 & 1.90 & 1.48 & 0.96 \\
\hline
\end{tabular}




\begin{tabular}{|c|c|c|c|c|c|c|c|c|c|c|c|c|c|}
\hline \multirow{2}{*}{$(\mathbf{n}, \mathbf{m})$} & \multirow{2}{*}{ Scheme } & \multicolumn{4}{|c|}{$\mathbf{T}=0.4$} & \multicolumn{4}{|c|}{$\mathbf{T}=0.6$} & \multicolumn{4}{|c|}{$\mathbf{T}=0.8$} \\
\hline & & LL & UL & HPD & CP & $\mathbf{L L}$ & UL & HPD & CP & LL & UL & HPD & CP \\
\hline & 6 & 0.23 & 2.26 & 2.03 & 0.92 & 0.32 & 2.09 & 1.76 & 0.94 & 0.36 & 1.92 & 1.56 & 0.93 \\
\hline \multirow[t]{6}{*}{$(50,10)$} & 1 & 0.38 & 1.80 & 1.42 & 0.93 & 0.38 & 1.79 & 1.41 & 0.95 & 0.48 & 1.81 & 1.33 & 0.96 \\
\hline & 2 & 0.11 & 2.71 & 2.60 & 0.94 & 0.20 & 2.19 & 1.98 & 0.91 & 0.29 & 1.98 & 1.69 & 0.94 \\
\hline & 3 & 0.36 & 1.81 & 1.45 & 0.91 & 0.48 & 1.83 & 1.35 & 0.94 & 0.48 & 1.81 & 1.33 & 0.96 \\
\hline & 4 & 0.37 & 1.88 & 1.51 & 0.94 & 0.46 & 1.78 & 1.33 & 0.94 & 0.48 & 1.82 & 1.34 & 0.95 \\
\hline & 5 & 0.32 & 2.00 & 1.68 & 0.92 & 0.40 & 1.96 & 1.56 & 0.94 & 0.41 & 1.85 & 1.45 & 0.94 \\
\hline & 6 & 0.25 & 2.19 & 1.95 & 0.93 & 0.33 & 2.07 & 1.73 & 0.94 & 0.39 & 1.98 & 1.60 & 0.94 \\
\hline \multirow[t]{6}{*}{$(30,15)$} & 1 & 0.26 & 1.98 & 1.72 & 0.93 & 0.42 & 1.67 & 1.25 & 0.91 & 0.53 & 1.60 & 1.08 & 0.94 \\
\hline & 2 & 0.16 & 2.41 & 2.25 & 0.92 & 0.28 & 1.99 & 1.70 & 0.93 & 0.37 & 1.77 & 1.40 & 0.92 \\
\hline & 3 & 0.24 & 2.03 & 1.79 & 0.92 & 0.40 & 1.79 & 1.39 & 0.94 & 0.47 & 1.65 & 1.18 & 0.93 \\
\hline & 4 & 0.25 & 1.96 & 1.71 & 0.94 & 0.44 & 1.71 & 1.28 & 0.93 & 0.52 & 1.63 & 1.13 & 0.94 \\
\hline & 5 & 0.26 & 2.00 & 1.74 & 0.94 & 0.41 & 1.79 & 1.38 & 0.92 & 0.47 & 1.72 & 1.24 & 0.95 \\
\hline & 6 & 0.21 & 2.18 & 1.97 & 0.93 & 0.33 & 1.90 & 1.58 & 0.94 & 0.42 & 1.80 & 1.38 & 0.94 \\
\hline \multirow[t]{6}{*}{$(40,15)$} & 1 & 0.32 & 1.81 & 1.50 & 0.94 & 0.49 & 1.61 & 1.12 & 0.95 & 0.56 & 1.62 & 1.06 & 0.95 \\
\hline & 2 & 0.14 & 2.30 & 2.16 & 0.92 & 0.29 & 1.98 & 1.69 & 0.92 & 0.39 & 1.80 & 1.42 & 0.93 \\
\hline & 3 & 0.30 & 1.88 & 1.57 & 0.95 & 0.47 & 1.68 & 1.21 & 0.95 & 0.54 & 1.61 & 1.07 & 0.94 \\
\hline & 4 & 0.32 & 1.81 & 1.49 & 0.93 & 0.49 & 1.65 & 1.16 & 0.94 & 0.49 & 1.70 & 1.21 & 0.95 \\
\hline & 5 & 0.31 & 1.87 & 1.56 & 0.93 & 0.46 & 1.78 & 1.32 & 0.93 & 0.50 & 1.74 & 1.24 & 0.95 \\
\hline & 6 & 0.25 & 2.18 & 1.93 & 0.92 & 0.36 & 1.91 & 1.55 & 0.94 & 0.42 & 1.75 & 1.33 & 0.93 \\
\hline \multirow[t]{6}{*}{$(50,15)$} & 1 & 0.37 & 1.73 & 1.36 & 0.95 & 0.53 & 1.60 & 1.07 & 0.94 & 0.56 & 1.61 & 1.05 & 0.95 \\
\hline & 2 & 0.16 & 2.40 & 2.24 & 0.93 & 0.29 & 1.99 & 1.69 & 0.93 & 0.39 & 1.79 & 1.41 & 0.94 \\
\hline & 3 & 0.35 & 1.77 & 1.42 & 0.93 & 0.51 & 1.62 & 1.11 & 0.94 & 0.56 & 1.61 & 1.05 & 0.94 \\
\hline & 4 & 0.37 & 1.73 & 1.36 & 0.94 & 0.52 & 1.62 & 1.10 & 0.93 & 0.56 & 1.63 & 1.07 & 0.95 \\
\hline & 5 & 0.36 & 1.83 & 1.46 & 0.95 & 0.47 & 1.75 & 1.28 & 0.94 & 0.50 & 1.69 & 1.19 & 0.96 \\
\hline & 6 & 0.27 & 2.13 & 1.86 & 0.93 & 0.38 & 1.92 & 1.55 & 0.93 & 0.44 & 1.78 & 1.34 & 0.94 \\
\hline \multirow[t]{6}{*}{$(40,20)$} & 1 & 0.31 & 1.80 & 1.48 & 0.93 & 0.49 & 1.58 & 1.09 & 0.93 & 0.58 & 1.50 & 0.92 & 0.94 \\
\hline & 2 & 0.19 & 2.15 & 1.96 & 0.89 & 0.34 & 1.82 & 1.48 & 0.93 & 0.44 & 1.68 & 1.23 & 0.93 \\
\hline & 3 & 0.30 & 1.87 & 1.57 & 0.94 & 0.46 & 1.65 & 1.20 & 0.94 & 0.54 & 1.57 & 1.03 & 0.94 \\
\hline & 4 & 0.32 & 1.82 & 1.50 & 0.94 & 0.48 & 1.57 & 1.09 & 0.92 & 0.58 & 1.52 & 0.94 & 0.95 \\
\hline & 5 & 0.32 & 1.83 & 1.51 & 0.94 & 0.47 & 1.68 & 1.20 & 0.93 & 0.53 & 1.61 & 1.08 & 0.94 \\
\hline & 6 & 0.26 & 2.05 & 1.80 & 0.91 & 0.39 & 1.82 & 1.43 & 0.94 & 0.47 & 1.67 & 1.20 & 0.93 \\
\hline \multirow[t]{6}{*}{$(50,20)$} & 1 & 0.37 & 1.73 & 1.36 & 0.93 & 0.54 & 1.53 & 0.99 & 0.94 & 0.61 & 1.52 & 0.91 & 0.93 \\
\hline & 2 & 0.19 & 2.09 & 1.90 & 0.88 & 0.33 & 1.78 & 1.45 & 0.92 & 0.45 & 1.67 & 1.23 & 0.92 \\
\hline & 3 & 0.36 & 1.79 & 1.43 & 0.94 & 0.52 & 1.60 & 1.08 & 0.95 & 0.59 & 1.53 & 0.94 & 0.95 \\
\hline & 4 & 0.37 & 1.72 & 1.35 & 0.92 & 0.53 & 1.53 & 1.00 & 0.93 & 0.59 & 1.49 & 0.90 & 0.95 \\
\hline & 5 & 0.38 & 1.80 & 1.42 & 0.95 & 0.50 & 1.67 & 1.17 & 0.94 & 0.54 & 1.60 & 1.06 & 0.96 \\
\hline & 6 & 0.29 & 2.04 & 1.75 & 0.92 & 0.39 & 1.77 & 1.38 & 0.93 & 0.49 & 1.69 & 1.21 & 0.94 \\
\hline \multirow[t]{6}{*}{$(50,25)$} & 1 & 0.36 & 1.71 & 1.35 & 0.94 & 0.54 & 1.52 & 0.99 & 0.95 & 0.63 & 1.45 & 0.82 & 0.95 \\
\hline & 2 & 0.24 & 2.04 & 1.80 & 0.92 & 0.40 & 1.75 & 1.35 & 0.93 & 0.50 & 1.61 & 1.11 & 0.93 \\
\hline & 3 & 0.35 & 1.77 & 1.42 & 0.92 & 0.52 & 1.61 & 1.09 & 0.95 & 0.58 & 1.51 & 0.92 & 0.95 \\
\hline & 4 & 0.36 & 1.72 & 1.35 & 0.94 & 0.53 & 1.51 & 0.98 & 0.94 & 0.62 & 1.45 & 0.83 & 0.93 \\
\hline & 5 & 0.37 & 1.74 & 1.37 & 0.95 & 0.53 & 1.59 & 1.06 & 0.94 & 0.57 & 1.54 & 0.96 & 0.94 \\
\hline & 6 & 0.29 & 1.94 & 1.65 & 0.93 & 0.42 & 1.69 & 1.28 & 0.93 & 0.51 & 1.59 & 1.08 & 0.94 \\
\hline
\end{tabular}

\section{Real data illustration}

In this section, we have considered a real data set from Lawless (2011) representing the survival times for ball bearings. The data given arose in tests on endurance of deep groove ball bearings. The data are the number of million revolutions before failure for each of the 23 ball bearings in the life tests and these are:

$17.88,28.92,33.00,41.52,42.12,45.60,48.48,51.84,51.96,54.12,55.56,67.80,68.64,68.64$, $68.88,84.12,93.12,98.64,105.12,105.84,127.92,128.04,173.40$

Raqab and Madi (2011) indicated that the one-parameter Rayleigh distribution provides a satisfactory fit to the given data set. We are assuming that the result of a random experiment is reported with some imprecision and may be represented by fuzzy numbers $\widetilde{x}_{i}=\left(a_{i}, b_{i}, c_{i}\right) ; i=1,2, \ldots, k$ with membership function given as:

$$
\xi_{\widetilde{x}_{i}}(x)=\left\{\begin{array}{cl}
\frac{x-\left(x_{i}-h\right)}{h} ; & x_{i}-h \leq x \leq x_{i} \\
\frac{\left(x_{i}+h\right)-x}{h} ; & x_{i} \leq x \leq x_{i}+h \\
0 \quad ; & \text { otherwise }
\end{array}\right.
$$

Where $h=0.05 x_{i}$. It is worthwhile to mention here that for illustration of our methodology 
Table 5: Average Bayes Estimate of the parameter of Rayleigh distribution based on TypeII progressive hybrid censored fuzzy lifetime data along with corresponding MSE's for fixed value of parameter $\vartheta=1, T=0.6, n=40, m=15$ and with varying prior and censoring scheme

\begin{tabular}{cccccccc}
\hline Prior & Scheme & Estimate & MSE & LL & UL & HPD & CP \\
\hline 1 & 1 & 1.0089 & 0.0689 & 0.52 & 1.54 & 1.03 & 0.96 \\
& 2 & 1.0346 & 0.1017 & 0.36 & 1.80 & 1.44 & 0.97 \\
& 3 & 1.0334 & 0.0769 & 0.50 & 1.61 & 1.11 & 0.95 \\
& 4 & 1.0178 & 0.0716 & 0.51 & 1.57 & 1.05 & 0.94 \\
& 5 & 1.0622 & 0.0951 & 0.50 & 1.68 & 1.19 & 0.95 \\
& 6 & 1.0591 & 0.1007 & 0.42 & 1.77 & 1.35 & 0.96 \\
\hline 2 & 1 & 1.0136 & 0.1014 & 0.49 & 1.59 & 1.11 & 0.93 \\
& 2 & 1.0408 & 0.2188 & 0.28 & 1.93 & 1.65 & 0.91 \\
& 3 & 1.0493 & 0.1106 & 0.47 & 1.68 & 1.21 & 0.93 \\
& 4 & 1.0476 & 0.1048 & 0.50 & 1.65 & 1.15 & 0.94 \\
& 5 & 1.0848 & 0.1463 & 0.46 & 1.78 & 1.32 & 0.93 \\
& 6 & 1.0821 & 0.1794 & 0.36 & 1.91 & 1.55 & 0.95 \\
\hline 3 & 1 & 1.0245 & 0.1068 & 0.49 & 1.61 & 1.12 & 0.93 \\
& 2 & 1.0349 & 0.2254 & 0.27 & 1.93 & 1.66 & 0.91 \\
& 3 & 1.0361 & 0.1126 & 0.46 & 1.67 & 1.21 & 0.93 \\
& 4 & 1.0369 & 0.1117 & 0.49 & 1.64 & 1.15 & 0.93 \\
& 5 & 1.0846 & 0.1561 & 0.46 & 1.78 & 1.32 & 0.93 \\
& 6 & 1.0740 & 0.1884 & 0.35 & 1.89 & 1.54 & 0.93 \\
\hline
\end{tabular}

we will censor the original data set. We have consider censored sample of size $m=11$ and seven different removal schemes.

Scheme 1: $R_{1}=R_{2}=\cdots=R_{10}=0$ and $R_{11}=12$;

Scheme 2: $R_{1}=12$ and $R_{2}=R_{3}=\cdots=R_{11}=0$;

Scheme 3: $R_{1}=R_{2}=\cdots=R_{10}=1$ and $R_{m}=2$;

Scheme 4: $R_{1}=R_{2}=\cdots=R_{6}=0, R_{7}=R_{8}=\cdots=R_{10}=2$ and $R_{11}=4$;

Scheme 5: $R_{1}=\cdots=R_{3}=0=R_{9}=\cdots=R_{11}$ and $R_{4}=R_{5}=2=R_{7}=R_{8}, R_{6}=4$

Scheme 6: $R_{1}=4, R_{2}=\cdots=R_{5}=2$ and $R_{6}=R_{7}=\cdots=R_{11}=0$

Also, maximum time of termination of experiment is prefixed as $T=68$. For illustration purpose lifetime of fail ball bearings, number of removals after each failure and lifetime of removed unit of the scheme 7 are given in Table 6 .

Table 6: Life times of ball bearings and removal scheme 7

\begin{tabular}{|c|ccc|c|ccc|}
\hline S.N. & $\begin{array}{c}\text { Lifetime of } \\
\text { failed unit } \\
(\mathbf{x})\end{array}$ & $\begin{array}{c}\text { Number of } \\
\text { removals } \\
(\mathbf{R})\end{array}$ & $\begin{array}{c}\text { Lifetime of } \\
\text { removed units } \\
(\mathbf{z})\end{array}$ & S.N. & $\begin{array}{c}\text { Lifetime of } \\
\text { failed unit } \\
(\mathbf{x})\end{array}$ & $\begin{array}{c}\text { Number of } \\
\text { removals } \\
(\mathbf{R})\end{array}$ & $\begin{array}{c}\text { Lifetime of } \\
\text { removed units } \\
(\mathbf{z})\end{array}$ \\
\hline 1. & 17.88 & 2 & $68.88,93.12$ & 7. & 48.48 & 1 & 68.64 \\
2. & 28.92 & 0 & - & 8. & 51.84 & 0 & - \\
3. & 33.00 & 1 & 55.56 & 9. & 67.80 & 2 & $105.84,173.40$ \\
4. & 41.52 & 2 & $54.12,127.92$ & 10. & 84.12 & 0 & - \\
5. & 42.12 & 0 & - & 11. & 98.64 & 2 & $105.12,128.04$ \\
6. & 45.60 & 2 & $51.96,68.64$ & & & & \\
\hline
\end{tabular}

We have obtained the MLE and MME by choosing suitable initial value of the parameter. To obtain the Bayes estimate non-informative prior is considered because enough prior information is not available. Average length of the HPD interval is also calculated. The ergodic mean, posterior samples, posterior density, and histogram corresponding to the removal scheme 7 are plotted in Figure 1 which shows that posterior of parameter $\vartheta$ is positively skewed, samples are well mixed and after burn-in period ergodic mean of generated samples become nearly constant. All results are summarized in Table 7. 
Table 7: MLE, MME, CAE and Bayes estimate of parameter $\vartheta$ along with its HPD interval for real data set.

\begin{tabular}{cccccc}
\hline Scheme & MLE & MME & CAE & BE & HPD \\
\hline 1 & 0.000188 & 0.000172 & 0.000192 & 0.000187 & 0.000214 \\
2 & 0.000188 & 0.000174 & 0.000195 & 0.000187 & 0.000268 \\
3 & 0.000166 & 0.000157 & 0.000168 & 0.000166 & 0.000205 \\
4 & 0.000157 & 0.000149 & 0.000159 & 0.000156 & 0.000187 \\
5 & 0.000137 & 0.000130 & 0.000140 & 0.000135 & 0.000180 \\
6 & 0.000090 & 0.000086 & 0.000089 & 0.000088 & 0.000142 \\
7 & 0.000160 & 0.000152 & 0.000163 & 0.000159 & 0.000199 \\
\hline
\end{tabular}

\section{Conclusion}

In this paper, we have considered the problem of estimation in the presence of Type-II progressive hybrid censored fuzzy sample. General procedure to obtain the estimators are provided and then Rayleigh distribution is used to validate the results. We have found that Bayesian procedure provides best estimate of the unknown parameter of the Rayleigh model with the smallest MSE among all the four estimators and it is followed by the MLE, MME and CAE. Average width of HPD intervals and coverage probabilities are also calculated and compared. Besides this, another finding of this study is that the estimates based on non-informative prior is less precise than that based on informative prior. It is seen that the removal scheme has an effect on the performance of estimators. Hence, if it is possible to control the removals, scheme with late removals should be chosen. However, the developed method can be used with any removal scheme. The methodology developed in this paper is quite flexible since it can be used with any distribution and will be very useful to the researchers, engineers, statisticians and in the field of medical where such type of fuzzy data is observed. Application of the developed methodology on Type-II progressive hybrid censored fuzzy data from another distribution could be a fruitful future research.

\section{References}

Balakrishnan N (2007). "Progressive Censoring Methodology: An Appraisal." Test, 16(2), 211-259.

Balakrishnan N, Aggarwala R (2000). Progressive Censoring: Theory, Methods, and Applications. Springer Science and Business Media.

Balakrishnan N, Cramer E (2014). "The Art of Progressive Censoring." Birkhauser, New York.

Balakrishnan N, Sandhu RA (1995). "A Simple Simulational Algorithm for Generating Progressive Type-II Censored Samples." The American Statistician, 49(2), 229-230.

Bhattacharya SK, Tyagi RK (1990). "Bayesian Survival Analysis Based on the Rayleigh Model." Trabajos de Estadistica, 5(1), 81-92.

Cai KY (2012). Introduction to Fuzzy Reliability, volume 363. Springer Science \& Business Media.

Childs A, Chandrasekar B, Balakrishnan N (2008). "Exact likelihood Inference for An Exponential Parameter Under Progressive Hybrid Censoring Schemes." In Statistical Models and Methods for Biomedical and Technical Systems, pp. 319-330. Springer. 
(a)

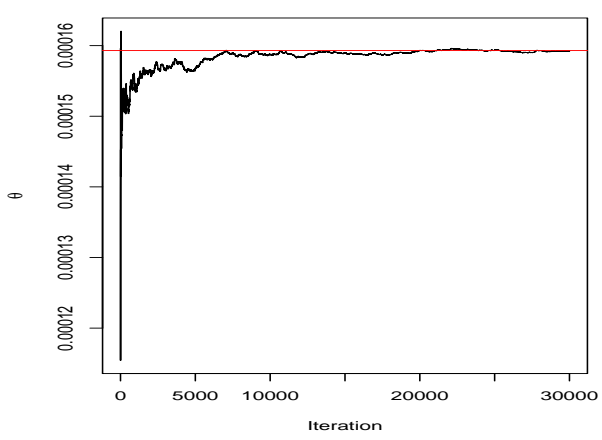

(c)

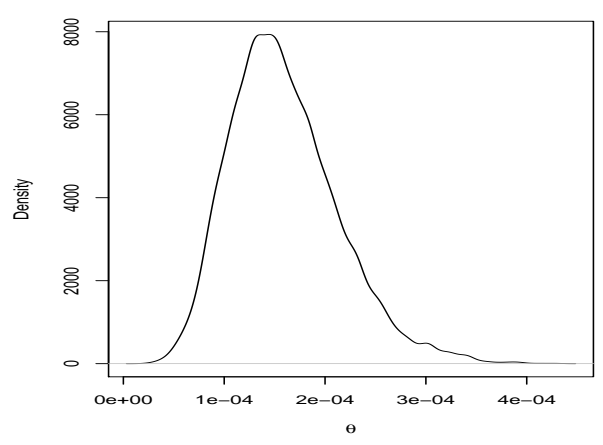

(b)

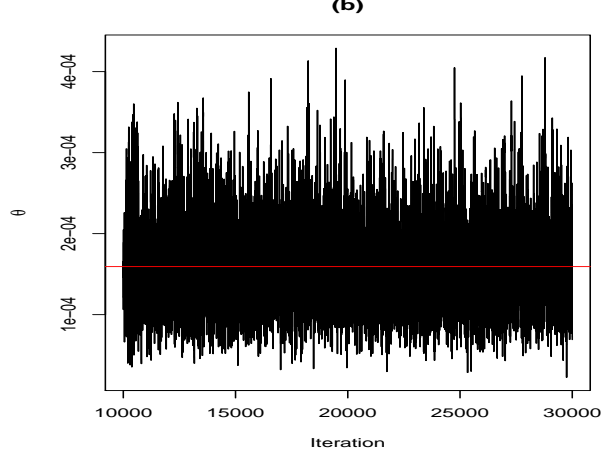

(d)

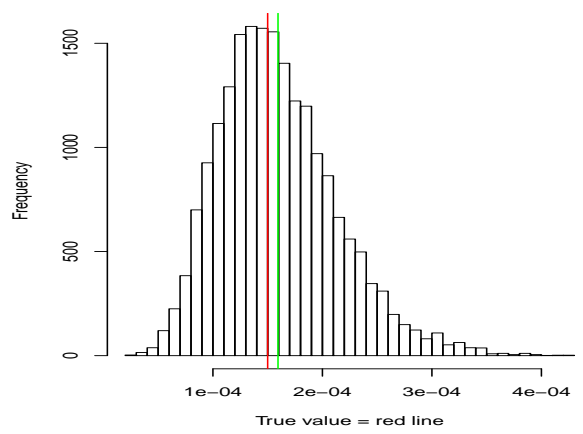

Figure 1: (a) Plot of ergodic Mean of simulated sample of $\vartheta$, (b) MCMC simulation runs (trace plot), (c) Kernel density estimate from simulated sample of $\vartheta$ and (d) Histogram of simulated sample of $\vartheta$ for real data set

Chung Y (1995). "Estimation of Scale Parameter from Rayleigh Distribution under Entropy Loss." Korean Journal of Computational and Applied Mathematics, 2(1), 33-40.

Cohen AC (1965). "Maximum Likelihood Estimation in the Weibull Distribution Based on Complete and on Censored Samples." Technometrics, 7(4), 579-588.

Cohen AC, Norgaard NJ (1977). "Progressively Censored Sampling in the Three-Parameter Gamma Distribution." Technometrics, 19(3), 333-340.

Davis HT, Feldstein ML (1979). "The Generalized Pareto Law as a Model for Progressively Censored Survival Data." Biometrika, 66(2), 299-306.

Dempster AP, Laird NM, Rubin DB (1977). "Maximum Likelihood from Incomplete Data via the EM Algorithm." Journal of the Royal Statistical Society. Series B (methodological), pp. $1-38$.

Denœux T (2011). "Maximum Likelihood Estimation from Fuzzy Data Using the EM Algorithm." Fuzzy sets and systems, 183(1), 72-91.

Dey S, Maiti SS (2012). "Bayesian Estimation of the Parameter of Rayleigh Distribution under the Extended JeffreyâĂŹs Prior." Electronic Journal of Applied Statistical Analysis, $\mathbf{5}(1), 44-59$.

Dubois D, Prade H (1998). "Possibility Theory: Qualitative and Quantitative Aspects." In Quantified Representation of Uncertainty and Imprecision, pp. 169-226. Springer.

Dubois DJ (1980). Fuzzy Sets and Systems: Theory and Applications, volume 144. Academic press.

Dyer DD, Whisenand CW (1973). "Best Linear Unbiased Estimator of the Parameter of the Rayleigh Distribution-Part I: Small Sample Theory for Censored Order Statistics." IEEE Transactions on Reliability, 22(1), 27-34. 
Hastings WK (1970). "Monte Carlo Sampling Methods using Markov Chains and their Applications." Biometrika, 57(1), 97-109.

Huang HZ, Zuo MJ, Sun ZQ (2006). "Bayesian Reliability Analysis for Fuzzy Lifetime Data." Fuzzy Sets and Systems, 157(12), 1674-1686.

Klir G, Yuan B (1995). Fuzzy Sets and Fuzzy Logic, volume 4. Prentice hall New Jersey.

Krishna H, Kumar K (2013). "Reliability Estimation in Generalized Inverted Exponential Distribution with Progressively Type-II Censored Sample." Journal of Statistical Computation and Simulation, 83(6), 1007-1019.

Kundu D (2007). "On Hybrid Censored Weibull Distribution." Journal of Statistical Planning and Inference, 137(7), 2127-2142.

Kundu D, Joarder A (2006). "Analysis of Type-II Progressively Hybrid Censored Data." Computational Statistics \& Data Analysis, 50(10), 2509-2528.

Kundu D, Joarder A, Krishna H (2009). "On Type-II Progressively Hybrid Censoring." Journal of Modern Applied Statistical Methods, 8(2), 18.

Lawless JF (2011). Statistical Models and Methods for Lifetime Data, volume 362. John Wiley \& Sons.

Liu X (2012). Survival Analysis: Models and Applications. John Wiley \& Sons.

Liu X, Li S (2012). "Cumulative Distribution Function Estimation with Fuzzy Data: Some Estimators and Further Problems." In SMPS, pp. 83-91. Springer.

Lu Y, Wang N (2013). "Method of Conditional Moments Based on Incomplete Data." International Journal of Mathematics \& Computationẩ̌ć, 20(3), 40-51.

McLachlan G, Krishnan T (2007). The EM Algorithm and Extensions, volume 382. John Wiley \& Sons.

Pak A, Chatrabgoun O (2016). "Inference for Exponential Parameter Under Progressive TypeII Censoring from Imprecise Lifetime." Electronic Journal of Applied Statistical Analysis, $\mathbf{9}(1), 227-245$.

Pak A, Parham GA, Saraj M (2013a). "Inference for the Weibull Distribution Based on Fuzzy Data." Revista Colombiana de Estadística, 36(2), 339-358.

Pak A, Parham GA, Saraj M (2013b). "On Estimation of Rayleigh Scale Parameter Under Doubly Type-II Censoring From Imprecise Data." Journal of Data Science, 11(2), 305-322.

Pak A, Parham GA, Saraj M (2014). "Inference for the Rayleigh Distribution Based on Progressive Type-II Fuzzy Censored Data." Journal of Modern Applied Statistical Methods, 13(1), 19.

Raqab MZ, Madi MT (2011). "Inference for the Generalized Rayleigh Distribution Based on Progressively Censored Data." Journal of Statistical Planning and Inference, 141(10), $3313-3322$.

Shafiq M, Viertl R (2015). "Empirical Reliability Functions Based on Fuzzy Life Time Data." Journal of Intelligent \& Fuzzy Systems, 28(2), 707-711.

Shafiq M, Viertl R (2017). "On the Estimation of Parameters, Survival Functions, and Hazard Rates based on Fuzzy Life Time Data." Communications in Statistics-Theory and Methods, 46(10), 5035-5055. 
Singh SK, Singh U, Kumar M (2013). "Estimation of Parameters of Generalized Inverted Exponential Distribution for Progressive Type-II Censored Sample with Binomial Removals." Journal of Probability and Statistics, 2013.

Singpurwalla ND, Booker JM (2004). "Membership Functions and Probability Measures of Fuzzy Sets." Journal of the American Statistical Association, 99(467), 867-877.

Viertl R (2009). "On Reliability Estimation based on Fuzzy Lifetime Data." Journal of Statistical Planning and Inference, 139(5), 1750-1755.

Viertl R (2011). Statistical Methods for Fuzzy Data. John Wiley \& Sons.

Viveros R, Balakrishnan N (1994). "Interval Estimation of Parameters of Life from Progressively Censored Data." Technometrics, 36(1), 84-91.

Zadeh LA (1965). "Fuzzy Sets." Information and Control, 8(3), 338-353.

Zadeh LA (1968). "Probability Measures of Fuzzy Events." Journal of Mathematical Analysis and Applications, 23(2), 421-427.

Zadeh LA (1975). "The Concept of a Linguistic Variable and its Application to Approximate ReasoningâĂŤI." Information Sciences, 8(3), 199-249.

Zimmermann HJ (2000). "An Application-Oriented View of Modeling Uncertainty." European Journal of Operational Research, 122(2), 190-198.

\author{
Affiliation: \\ Ankita Chaturvedi \\ Department of Statistics, Institute of Science \\ Banaras Hindu University \\ Varanasi, India-221005 \\ E-mail: ankita.c187@gmail.com
}

\section{Austrian Journal of Statistics}

published by the Austrian Society of Statistics

Volume 47

June 2018 http://www.ajs.or.at/

http://www.osg.or.at/

Submitted: 2017-10-31

Accepted: 2018-02-22 